\title{
Optical coherence tomography: fundamentals and clinical utility
}

\author{
Vijayakumar Subban ${ }^{1}$, Owen Christopher Raffel $^{2,3,4,5}$ \\ ${ }^{1}$ Institute of Cardiovascular Diseases, The Madras Medical Mission, Chennai, India; ${ }^{2}$ CardioVascular Clinics, St. Andrews War Memorial Hospital, \\ Queensland, Australia; ${ }^{3}$ Cardiology Program, The Prince Charles Hospital, Queensland, Australia; ${ }^{4}$ Queensland University of Technology, \\ Queensland, Australia; ${ }^{5}$ University of Queensland, Queensland, Australia \\ Contributions: (I) Conception and design: All authors; (II) Administrative support: All authors; (III) Provision of study materials or patients: All \\ authors; (IV) Collection and assembly of data: None; (V) Data analysis and interpretation: None; (VI) Manuscript writing: All authors; (VII) Final \\ approval of manuscript: All authors. \\ Correspondence to: Owen Christopher Raffel, MB, ChB, FRACP. Cardiology Program, The Prince Charles Hospital, Rode Road, Chermside, \\ Queensland 4032, Australia. Email: chris.raffel@qut.edu.au.
}

\begin{abstract}
Although coronary angiography is the standard method employed to assess the severity of coronary artery disease and to guide treatment strategies, it provides only 2D image of the intravascular lesions. In contrast, intravascular imaging modalities such as optical coherence tomography (OCT) produce cross-sectional images of the coronary arteries at a far greater spatial resolution, capable of accurately determining vessel size as well as plaque morphology, eliminating many of the disadvantages inherent to angiography. This review will discuss the role of OCT in the catherization laboratory for the assessment and management of coronary disease.
\end{abstract}

Keywords: Acute coronary syndromes (ACS); coronary artery disease (CAD); tomography, optical coherence

Submitted Feb 19, 2020. Accepted for publication Apr 20, 2020.

doi: $10.21037 / \mathrm{cdt}-20-253$

View this article at: http://dx.doi.org/10.21037/cdt-20-253

\section{Introduction}

Coronary angiography (CAG) remains the gold standard and most commonly used imaging modality for the invasive assessment of coronary artery disease (CAD) and for guiding percutaneous coronary intervention (PCI). However, it has several limitations including poor resolution, angle dependency, vessel overlap and foreshortening and significant observer variability in assessing coronary stenoses. Additional, CAG is a 2-dimensional luminogram and does not provide information about the vessel wall and plaque characteristics (1).

With their ability to produce high-resolution crosssectional images of the coronary arteries, intravascular imaging (IVI) modalities compliment CAG in the quantitative and qualitative assessment of CAD both for diagnostic and interventional purposes. There are four major IVI modalities currently available for coronary imaging, namely intravascular ultrasound (IVUS), optical coherence tomography (OCT), angioscopy and near infrared spectroscopy (NIRS).

The current review focuses on OCT, a light-based ultrahigh resolution IVI modality that provides images of exquisite detail.

\section{Fundamentals of OCT}

\section{Technology}

OCT is fundamentally an optical analog of IVUS using near infra-red light (instead of sound) to produce highresolution cross-sectional images of the coronary artery. OCT forms images by measuring the echo time delay and intensity of the backscattered optical signals from the various tissue interfaces of the vessel wall. However, the speed of light at $3 \times 10^{8} \mathrm{~m} / \mathrm{s}$ as compared to sound at $1,500 \mathrm{~m} / \mathrm{s}$ makes it impossible to directly detect the echo time delays of the reflected light waves and instead uses interferometric detection where the signals reflected off the tissue is compared with a fixed reference signal. The very high frequency of the light provides $10-15 \mu$ resolution but 

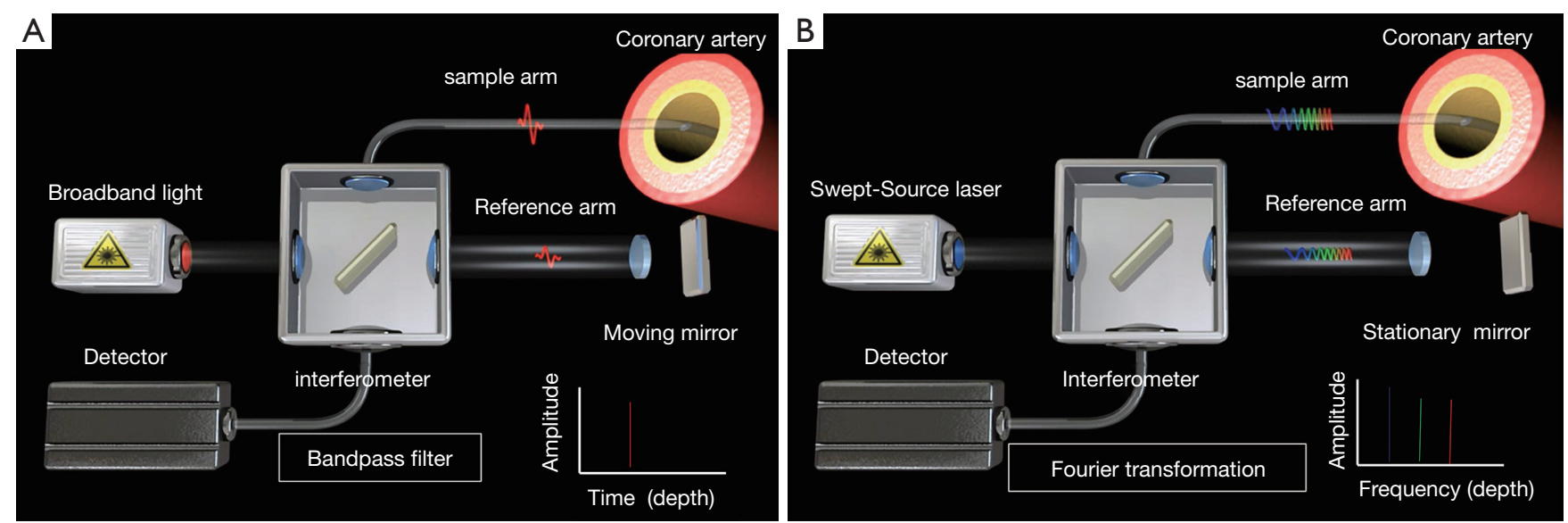

Figure 1 Types of OCT systems. (A) Time domain OCT system uses a broadband light source and a moving reference mirror. The interference signals with different time delays are captured sequentially that limits the rate of image acquisition and pull back speed (2-3 mm/s). (B) Frequency domain OCT system uses swept-source laser (wavelengths from 1,250 to 1,350 nm) and a fixed reference mirror. Fourier transformation is used to process the interference patterns at different wave lengths simultaneously to provide the amplitude and frequency profiles of the light signals reflected from different depths. This allows FD detector to measure all the signals that forms an A-line at the same time and rapid image acquisition (figures courtesy: Abbott Vascular). OCT, optical coherence tomography.

at the same time limits tissue penetration to $1-2 \mathrm{~mm}(2)$.

There are two types of OCT systems: time domain OCT (TD-OCT) and frequency domain OCT (FD-OCT). TDOCT technology was used in the first clinical OCT systems and is now of historical interest having been superseded by the faster FD-OCT technology (Figure 1). FD-OCT uses a narrow bandwidth light source that sweeps rapidly between wavelengths 1,250 to $1,350 \mathrm{~nm}$ and a fixed reference mirror. The interference patterns at different wavelengths are processed by Fourier transformation to provide the amplitude profile and time delay of the waves reflected from different depths. This enables FD detector to measure all echo time delays that form an A-line at the same time allowing significantly faster image acquisition rates than the original TD-OCT systems. The technology behind these different systems and procedural details for image acquisition have been described in detail elsewhere $(2,3)$.

\section{Image acquisition}

There are currently two commercially available IV OCT systems from Abbott (Optis FD-OCT system) and Terumo corporation (Lunawave Optical Frequency Domain Imaging system). Standalone and integrated systems are available. They both use monorail rapid exchange catheters compatible with standard 6-F guiding catheters. Pullback speeds up to $40 \mathrm{~mm} / \mathrm{s}$ and pullback lengths up to $150 \mathrm{~mm}$ are achievable depending on the system. Blood clearing is performed by contrast injection through the guide catheter without the need for balloon occlusion. Angiographic co-registration is possible with both systems and real-time $3 \mathrm{D}$ reconstruction with the Abbott Optis system (4). Where renal impairment limits the liberal use of contrast, alternatives such as dextran and Ringers lactate have been used successfully for blood clearing. Alternatively, a guide extension device such as a Guideliner placed close to the target area enables excellent contrast flushing using extremely small volumes.

\section{Qualitative OCT imaging: arterial wall and atherosclerosis}

Interaction of light photons with the tissues of the body occurs in the form of reflection, refraction, attenuation or scattering. When light photons encounter a tissue interface, part of them are reflected from the surface and part are allowed to pass through. The amount of reflection (Backscatter) depends on the change in refractory index at the interface and the angle of incidence. The amount of backscattering determines the brightness of the tissue in the image. In the case of strong reflectors (materials with high backscatter), all the signals may be reflected back from the surface and none of them pass through it, resulting in a bright interface with shadowing beyond the leading edge of the structure. Metal (stent struts and guide wires) is a strong reflector of light and causes shadowing. Calcium reflects light poorly and allows it to pass 

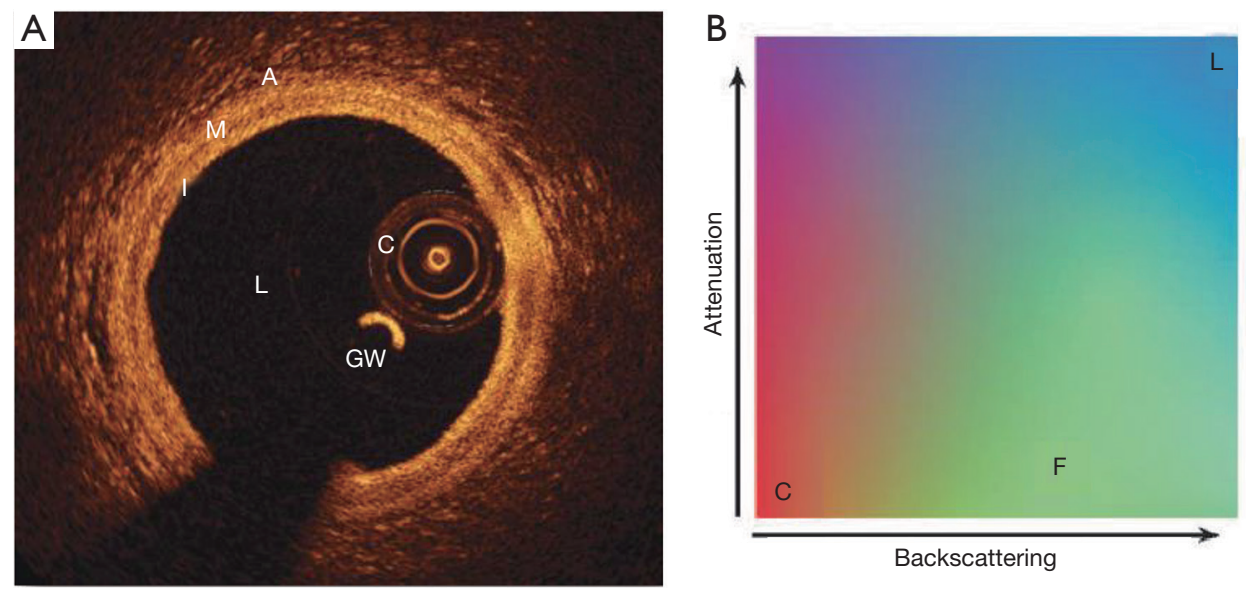

Figure 2 OCT appearance of normal coronary artery and color map of different tissue components. (A) Normal coronary artery on OCT imaging has 4 components. (I) Imaging catheter (C); (II) guide wire (GW); (III) echolucent lumen (L) and (IV) three-layered vessel wall (I: intima, M: media, A: adventitia). (B) Colormap combining backscattering and attenuation coefficients for calcific (C), fibrous (F) and lipid (L) tissue. OCT, optical coherence tomography. B modified from Xu et al. (5).

through. Hence, it is possible to measure the thickness of the calcium with OCT imaging. Attenuation is decrease in the signal intensity when the signals pass through the tissue and occurs mainly due to absorption by the tissue. Attenuation determines the penetration depth of the signals: strongly attenuating tissues have very low penetration depth and poor visibility of the deeper structures. Scattering is reflection in multiple directions and occurs with small moving structures, such as red blood cells and produces a typical appearance of speckling in the image. With OCT imaging, red blood cells cause intense scattering of the light that interferes with imaging and hence needs lumen clearance typically with contrast medium to clear the lumen of blood (2).

\section{Normal coronary artery}

A typical OCT image has 4 components. (I) The catheter: a circular structure inside the lumen. (II) The guide wire: it appears as a bright spot with a wedge-shaped shadow. (III) Echolucent lumen: In an optimally acquired OCT image, the lumen appears uniformly dark as the blood is cleared with contrast. (IV) The vessel wall: In a normal adult coronary artery, the vessel wall has a three-layered architecture. The intimal and adventitial layers have abundance of collagen and elastin. They reflect light waves strongly and appear bright. The media contains largely smooth muscle cells that poorly reflects light waves and appears dark. This gives the vessel wall a three-layered "bright-dark-bright" pattern (Figure 2A) (2).

\section{Characterization of atherosclerosis}

Intimal atherosclerotic plaque evolves through a number of stages that culminate either in plaque disruption with thrombosis or progressive luminal narrowing which may be asymptomatic or result in chronic stable angina. Contemporary classification of plaque morphology divides atherosclerotic lesions into 4 subtypes: (I) nonatherosclerotic intimal lesions (intimal thickening, intimal xanthoma); (II) progressive atherosclerotic lesions [pathological intimal thickening, fibroatheroma, intraplaque hemorrhage or plaque fissure, thin cap fibroatheroma (TCFA)]; (III) lesions with acute thrombi [plaque rupture (PR), plaque erosion (PE), calcified nodule (CN)]; (IV) healed lesions (healed $\mathrm{PR}, \mathrm{PE}$ or $\mathrm{CN})$. This classification is based on the ex vivo analysis of coronary specimens derived from patients who experienced sudden cardiac death or died from acute myocardial infarction (MI). In vivo characterization of these abnormalities may help in improving patient outcomes $(6,7)$.

OCT dramatically improves the assessment of coronary atherosclerotic lesions in clinical practice and is able to depict features characteristic of the different stages of 
atherosclerosis (2).

\section{Optical properties of basic plaque components and their resulting appearance on OCT Imaging}

\section{(I) Fibrous}

Fibrous tissue causes high backscattering and low attenuation of light and results in a bright signal-rich and homogenous appearance on OCT imaging (2) (Figure 2B).

\section{(II) Calcified tissue}

Calcified tissue results in low backscattering and low attenuation of the light source with the appearance of a signal poor (dark) region on OCT imaging. In addition, as calcium has a low-backscattering property compared to that of fibrous tissue (highly backscattering) the OCT signal drops suddenly at the borders between fibrous and calcified tissue resulting in sharply defined borders of the calcium deposits $(2,5)$.

\section{(III) Lipid/necrotic core}

Lipid has high backscattering properties similar to fibrous tissue so the anatomical border between fibrous tissue and the lipid pool cannot be distinguished based on image intensity alone. Indeed, the most superficial layers of lipid maybe brighter (more signal rich) than the adjacent fibrous cap. The fibrous-lipid borders therefore are diffuse and not well defined unlike calcium. In addition, lipid attenuates light much more than fibrous tissue so the light signal attenuates very fast, and the lipid pool becomes rapidly and progressively darker (signal poor) $(2,5)$.

\section{(IV) Macrophages/inflammatory cells}

Highly backscattering signal rich (bright) spots or bands with sharp signal shadowing, usually occurring at the interface between fibrous cap and necrotic core (2).

\section{Nonatherosclerotic intimal lesions}

Intimal thickening is characterized by areas of thickened intima constituted by gathering of smooth muscle cells in an extracellular matrix rich in proteoglycan and no inflammatory cells. Intimal xanthoma contains superficial macrophage foam cells in addition (6). OCT uses an arbitrary cut off value of $300-600 \mu$ of intimal thickness to differentiate nonatherosclerotic intimal changes from normal coronary artery $(<300 \mu)$ and atherosclerotic lesions $(>600 \mu)$. On OCT imaging, intimal thickening appears as homogeneous signal rich intima with a thickness between $300-600 \mu$ and visibility of media in $\geq 3$ quadrants (Figure $3 A$ ). In intimal xanthoma, foamy macrophages produce shadowing of the light (Figure $3 B)(7,8)$.

\section{Progressive atherosclerotic lesions}

\section{(I) Pathological intimal thickening}

Pathological intimal thickening is characterized by lesions with focal extracellular accumulation of acellular lipid adjacent to media and a luminal intima that contains smooth muscle cells embedded in a proteoglycan and collagen rich matrix. There may be accumulation of macrophages closer to the lumen (6).

\section{(II) Fibroatheromas}

Macrophage infiltration of the lipid pools causes progressive destruction of the extracellular matrix elements and formation of necrotic core that transforms pathological intimal thickening into a fibroatheroma. Early fibroatheromas are characterized by focal loss of extracellular matrix while late fibroatheromas by complete depletion of extracellular matrix with large amounts of free cholesterol and cellular debris. Both early and late fibroatheromas are covered by thick fibrous caps (6).

\section{(III) TCFAs}

TCFA are a specific type of fibroatheromas that contain a large necrotic core covered by a thin fibrous cap $(<65 \mu)$ with paucity of smooth muscle cells and large infiltrations of inflammatory cells. They are also called as "vulnerable plaques"-a precursor lesion for PR (6).

On OCT imaging, pathological intimal thickening is characterized by a homogenous, signal rich plaque characteristic of fibrous tissue (OCT defines pathological intimal thickening and fibrous plaque in a similar manner), $>600 \mu$ thickness and lipid or calcium when present, occupy less than one quadrant (Figure 3C).

OCT cannot differentiate lipid pool from necrotic core but with its almost microscopic resolution enables it to assess the overlying fibrous cap thickness (FCT). With OCT imaging, lipid pool/necrotic core appears as a progressively attenuating signal poor area with poorly defined boundaries and the overlying fibrous cap as a homogenous signal rich layer of tissue. As noted above the fibrous cap-lipid boundary in reality is not sharply defined but the lipid attenuates rapidly such that the measurements taken as FCT correlate highly with histopathological measurements (5). Fibroatheroma is defined as a plaque with $\geq 1$ quadrant of lipid pool/necrotic core and further classified based on fibrous cap thickness into thick cap fibroatheroma $(>65 \mu)$ and TCFA $(<65 \mu)$ (Figure 3D,E). Compared to early fibroatheromas, late fibroatheromas are associated with significant lumen compromise $(7,8)$. OCT imaging also identifies other features of fibroatheromas 

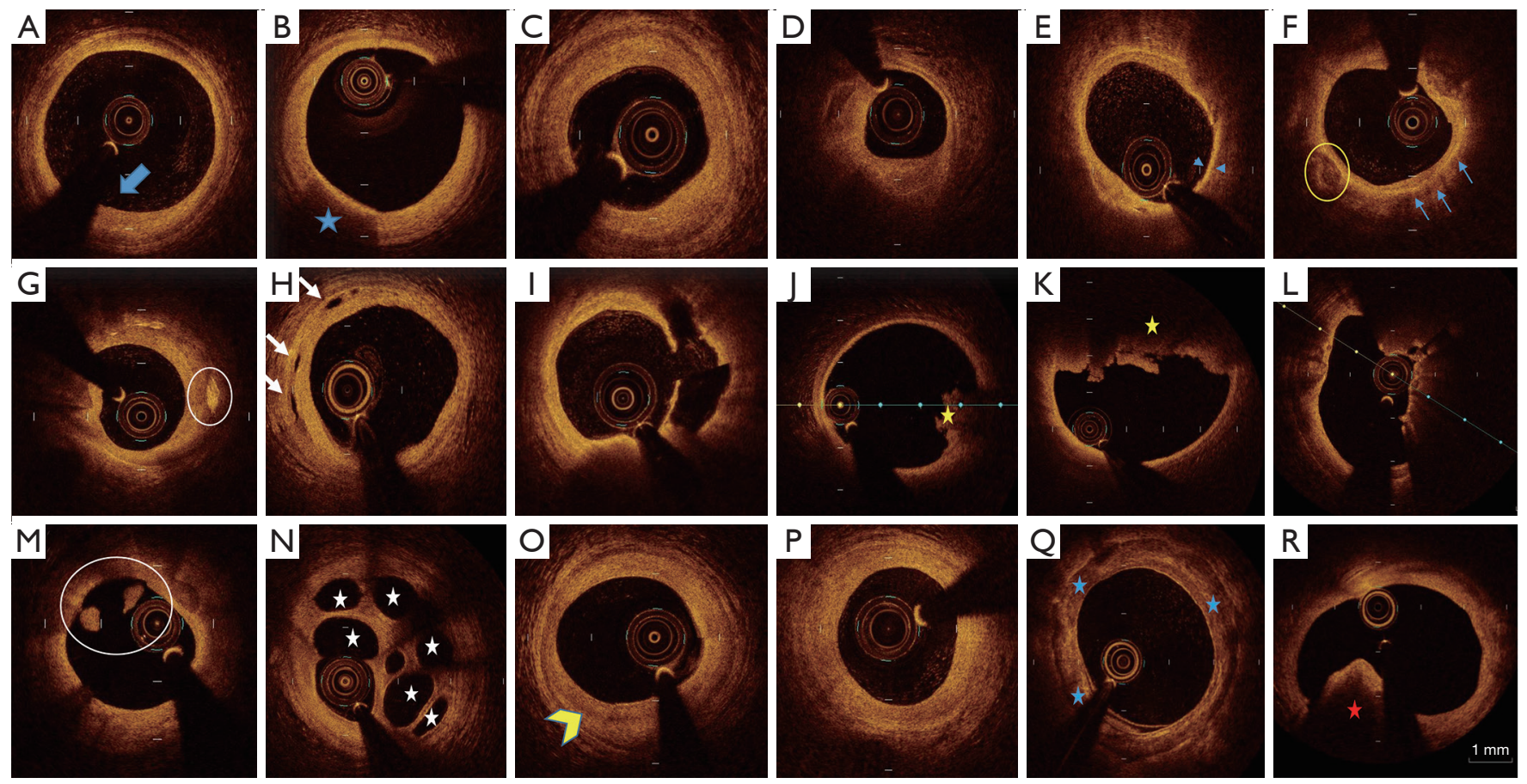

Figure 3 Types of atherosclerotic lesions. (A,B) Non-atherosclerotic intimal lesions: (A) intimal thickening (fibrous/fibro-fatty appearing tissue with thickness 300-600 $\mu$, arrows); (B) intimal xanthoma [signal-rich lumen border with deep signal attenuation and sharp lateral borders suggestive of superficial macrophage foam cells (star)]. (C,D,E) Progressive atherosclerotic lesions: (C) pathological intimal thickening (intimal thickness $>600 \mu$, lipid/necrotic core less than one quadrant). OCT imaging does not differentiate between pathological intimal thickening and fibrous plaque; (D) fibroatheroma (lipid/necrotic core more than one quadrant); (E) thin-cap fibroatheroma [Fibrous cap thickness $<65 \mu$, arrow heads]. (F,G,H) Features of vulnerability for rupture: $(\mathrm{F})$ macrophage accumulation (signal rich spots with shadowing, arrows) and spotty calcification (yellow circle); (G) cholesterol crystals [signal-rich streaks with low attenuation, white circle]; (H) microvessels [signal voids within the plaque, white arrow heads]. (I,J,K,L,M,N) Lesions with thrombus: (I) plaque rupture (fibrous cap rupture with underlying cavity at 3-o'clock position); (J) definitive erosion (intact fibrous cap with attached red thrombus, yellow star); (K) probable erosion [large red thrombus (intraluminal mass with high backscatter and high attenuation, yellow star) with no adjacent necrotic core/lipid or superficial calcium (not shown in the figure)]; (L) calcified nodule (eruptive calcification with thrombus, 2- to 5-o'clock position); (M) white thrombus (intraluminal mass with high backscatter and low attenuation, white circle); (N) recanalized thrombus (intraluminal mass with channels, white stars). (O,P,Q,R) Advanced lesions: $(\mathrm{O})$ healed plaque erosion (layered pattern with no evidence of underlying rupture, arrow head); (P) fibrous plaque (intimal thickness $>600 \mu$, homogeneous, high backscatter, low attenuation); (Q) circumferential calcium plate (heterogeneous, low backscatter, low attenuation, clear borders, blue stars); (R) nodular calcification (red star). OCT, optical coherence tomography.

such as macrophages, microvessels (intraplaque signal voids measuring 50-300 $\mu$ and extending over 3 or more frames), cholesterol crystals (signal rich streaks with low attenuation) and spotty calcification (small calcium deposit with an arc $<90^{\circ}$ ) (Figure 3F,G,H) (2).

\section{Lesions with acute thrombi}

There are 3 common underlying mechanisms of coronary thrombosis clinically manifesting as an acute coronary syndrome (ACS), namely PR, PE and CN. OCT identifies both $\mathrm{PR}$ and $\mathrm{CN}$ with high sensitivity. As the imaging of endothelium is beyond the resolution of current OCT systems, the detection of $\mathrm{PE}$ is a diagnosis of exclusion. Hence, it is also referred to as ACS with intact fibrous cap.

\section{(I) PR}

$\mathrm{PR}$ is the most common pathological mechanism of ACS and results from disruption of the fibrous cap overlying a TCFA. On OCT imaging, it is characterized by an intraplaque cavity communicating with the lumen and 
fibrous cap remnants (Figure 3I).

(II) $\mathbf{P E}$

$\mathrm{PE}$ is the second most common mechanism resulting in an ACS and occurs due to disruption of the endothelial layer covering the plaque. There is no evidence of a ruptured fibrous cap and usually the absence of a significant lipid pool or necrotic core. The underlying plaque type is either pathological intimal thickening or early fibroatheroma. OCT defined PE is classified into two types: definite erosion-thrombus attached to a visualized intact fibrous cap (Figure 3f) and probable erosion-irregular lumen surface with no thrombus or luminal thrombus in the absence of adjacent superficial calcium or lipid/necrotic core when the underlying plaque is not visible (Figure $3 K$ ).

(III) $\mathrm{CNs}$

$\mathrm{CN}$ is the least common cause of coronary thrombosis and usually occurs in the background of severe coronary calcification. Thrombosis occurs when nodular calcification breaches the overlying fibrous cap. On IVUS/OCT imaging it appears as protruding nodular calcification with dorsal shadowing (Figure $3 L)(6,7,9)$.

\section{(IV) Thrombus}

OCT imaging clearly differentiates red thrombus from white thrombus. Red thrombus appears as an intraluminal mass with high backscatter and high attenuation (Figure $3 \mathrm{~K}$ ) whereas white thrombus appears as an intraluminal mass with high backscatter and low attenuation (Figure 3M) (2). A recanalized thrombus produces a lotus root or Swiss cheese appearance (Figure 3N) (10).

\section{Advanced atherosclerotic lesions}

Although the early atherosclerotic lesions are more prevalent in the real world, the interventionist commonly encounter advanced atherosclerotic lesions in the catheterization laboratory and are better characterized with current IVI modalities compared to early lesions.

\section{(I) Healed lesions}

Episodes of coronary thrombosis from PR, PE or CN are common and manifest with an ACS only when the thrombus is either acutely occlusive or embolizes distally, otherwise, they remain silent. This non-occlusive thrombus progressively organizes and heals and is the primary contributor to lesion progression. Healed lesions on OCT typically produce a layered pattern of tissue with different optical densities with or without underlying necrotic core or calcification (Figure 3O) $(6,7)$. When silent thrombosis is occlusive, it evolves into chronic total occlusion (CTO). Early CTO lesions are characterized by plaques with large necrotic core, organizing thrombi and less negative remodeling in comparison to more fibrous or fibrocalcific appearing tissue with severe negative remodeling in older CTO lesions (11).

\section{(II) Fibrous plaque}

The non-occlusive thrombus formed during these silent episodes also propagates both proximally and distally. When this propagating thrombus heals, it results in formation of fibrous plaques. On OCT imaging fibrous plaque are defined as a homogenous, signal rich plaque with low signal attenuation and $>600 \mu$ thickness (Figure $3 P$ ) $(2,8)$.

\section{(III) Fibrocalcific plaque/nodular calcification}

Calcification begins to appear in the early progressive atherosclerotic lesions. It usually occurs in the form of microcalcification that results from apoptosis of the smooth muscle cells and macrophages. In the advanced lesions, the plaque components, such as collagen, proteoglycan and necrotic core evolve into large calcium deposits. These deposits usually occur in the form calcium sheets/ plates and are closer to media. In elderly individuals with tortuous arteries, these calcium plates fracture to form nodular calcification surrounded by fibrin deposits (6). On OCT imaging, calcium appears as a heterogeneous area of low backscatter with low attenuation and clear borders surrounded by fibrous tissue (Figure 3Q). Nodular calcium appears similar to $\mathrm{CN}$ but with intact fibrous cap and no luminal thrombus (Figure 3R) (2).

\section{Quantitative OCT imaging}

OCT provides accurate cross sectional and longitudinal measurements. The OCT system is calibrated to the imaging catheter before measurements. In a native vessel, there are two interfaces: lumen-intima and the mediaadventitia (external elastic membrane, EEL) while there are 3 interfaces in a stented vessel: lumen-neointima, stent and EEL. In view of its higher accuracy and reproducibility, it is recommended to make all the measurements from the leading edge to leading edge of the interfaces. The diameter measurements are done through the center of the lumen. Length is estimated from the automatic pullback (pull back speed $x$ duration of pull back). The common terminologies and measurements are summarized in Table 1 (2).

The clear demarcation of the lumen-intima interface with OCT makes automated measurements feasible and highly accurate. In addition, the newer lumen profile function provides graphic display (like an ideal angiographic view) 
Table 1 Common OCT measurements

Pre-PCl measurements

Vessel/external elastic membrane (EEM) area: area bounded by the media-adventitia interface

Lumen CSA: area circumscribed by the lumen border

Minimum lumen area (MLA): smallest CSA in the lesion segment

Maximum and minimum EEM diameters: the longest and shortest EEM distances measured through the center of the lumen

Maximum and minimum lumen diameters: the longest and shortest lumen distances measured through the center of the lumen

Proximal and distal reference: the sites with largest lumen areas on either side of the lesion within $10 \mathrm{~mm}$ and in the same segment

Plaque + media area $=$ EEM CSA - lumen CSA

Plaque burden $=($ EEM CSA - lumen CSA $) / E E M ~ C S A ~ \times 100$

Lumen area stenosis: (the reference lumen CSA - minimal lumen CSA)/reference lumen CSA ×100

Post stenting measurements

Stent CSA: area circumscribed by the stent border

Proximal and distal reference: the sites with largest lumen areas on either side of the stent and within $5 \mathrm{~mm}$ of the edges

Maximum (Max) and minimum (Min) stent diameters (SD): the longest and shortest diameters measured through the center of the stent

Minimum stent CSA $=$ smallest stent CSA

Stent expansion: minimum stent CSA in correlation with reference lumen CSA (proximal, distal or average)

Stent symmetry $=($ Max SD - Min SD $) /$ Max SD

Neointimal area $=$ Stent CSA - lumen CSA

In-stent lumen area stenosis $=($ the reference lumen CSA - minimal lumen in-stent CSA)/reference lumen CSA $\times 100$

Maximum and minimum neointimal thickness: the longest and shortest dimensions between the lumen border of the neointima to the adluminal stent border along the line passing through the center of the lumen

$\%$ neo intimal area $=$ Neointimal CSA/stent CSA

OCT, optical coherence tomography; PCI, percutaneous coronary intervention; CSA, cross-sectional area.

of frame by frame lumen measurements over the entire pull back length. This enables precise understanding of the vessel size, disease extent and vessel tapering. Moreover, it automatically locates the frame with minimum lumen area (MLA) and once the correct reference vessel frames are manually located, the system automatically provides the \% area/diameter stenosis, reference vessel dimensions and the lesion length to facilitate device sizing (Figure 4) (4).

While measurements with OCT are highly correlated with the reference standard of IVUS, there are some differences. The OPUS-CLASS study compared lumen dimensions measured with IVUS and OCT both in phantom model and human coronary arteries. In the phantom model, OCT area matched actual dimension of the phantom $\left(7.45 \pm 0.17 v s .7 .45 \mathrm{~mm}^{2}\right)$ and IVUS area was larger by $8 \%\left(8.03 \pm 0.58 \mathrm{~mm}^{2} ; \mathrm{P}<0.001\right)$. In the human study, OCT measured minimum lumen diameter (MLD) and MLA were smaller by $9 \%(1.91 \pm 0.69$ vs. $2.09 \pm 0.60 \mathrm{~mm}$; $\mathrm{P}<0.001)$ and $10 \%\left(3.68 \pm 2.06\right.$ vs. $\left.3.27 \pm 2.22 \mathrm{~mm}^{2} ; \mathrm{P}<0.001\right)$ respectively when compared to IVUS measurements (4). This is most likely because the vessel wall-lumen interface is much better defined with OCT compared to IVUS and that IVUS measurements are made in diastole where coronary filling is maximal and coronary luminal dimensions largest. Based on these observations, the recent OCT studies have used newer stent sizing algorithms to match post stent measurements with that of IVUS guided stent sizing.

\section{Artifacts in OCT imaging}

Artifacts are common with OCT imaging. They may adversely affect the image quality that limits identification 


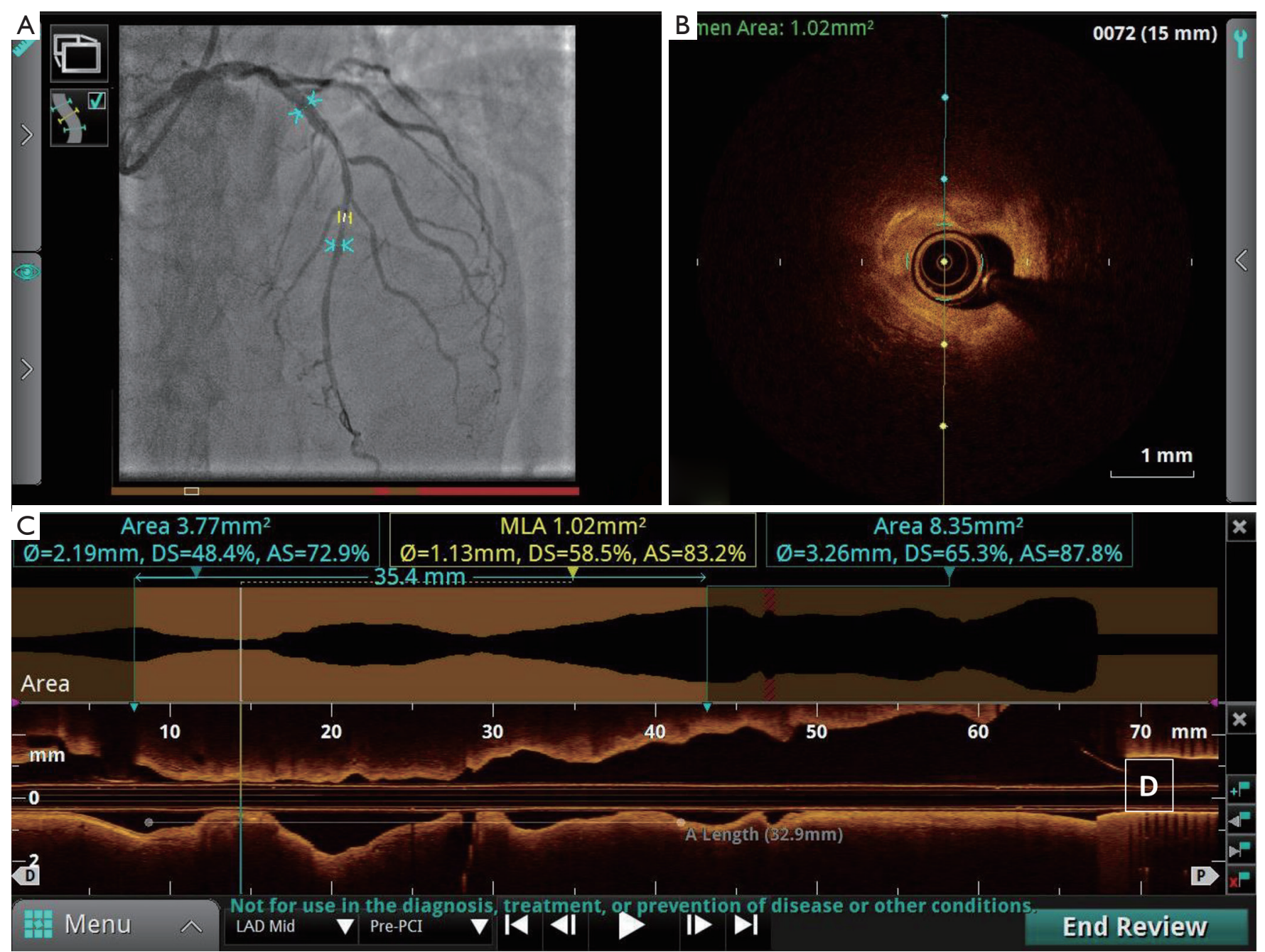

Figure 4 OCT monitor screen display. (A) Angiogram marked with references [blue markers] and minimal lumen area (yellow marker); (B) cross sectional image; (C) lumen profile with automatic measurement and display of proximal and distal references (blue boxes), minimal lumen area (yellow box), and lesion length (blue line); (D) long view. OCT, optical coherence tomography.

of the true tissue structures or sometimes be misinterpreted as pathologies that leads to unnecessary interventions (12).

\section{Nonuniform rotational distortion (NURD)}

NURD is unique to mechanical imaging systems. It results from uneven drag or friction on the drive cable that perturbs its smooth rotation. It appears as smearing or geometric distortion in the image that conceals the structures in that part of the circumference and limits accurate cross-sectional measurements. It is often due to tortuosity or acute bends in the artery, tight stenosis, heavy calcification, small guiding catheter, guiding catheter with multiple curves, excessive tightening of the hemostatic valve or defective imaging catheter (Figure 5A) $(2,12)$.

\section{Sew-up artifacts}

Rapid movement of the artery or the imaging catheter during the acquisition of a single cross-sectional image causes malalignment along the circumference of the image which is known as sew-up/seamline artifact (Figure 5A) (2).

\section{Obliquity}

Cross-sectional imaging of the vessel occurs perpendicular 

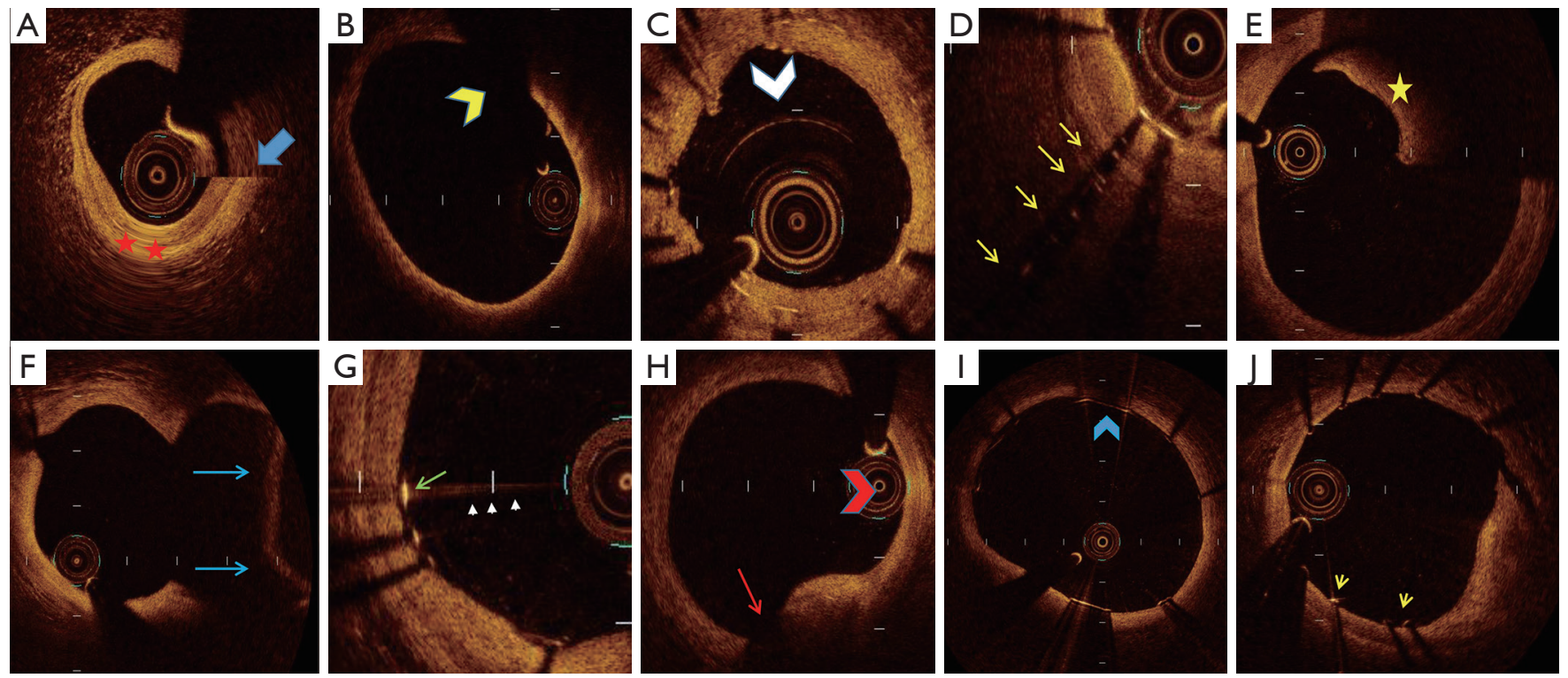

Figure 5 OCT artifacts. (A) Non-uniform rotational deformity (red stars) and sew up artifact (blue arrow); (B) obliquity artifact, yellow arrow head indicates shadowing artifact from guide wire; (C) multiple reflection artifact (white arrow head); (D) stent reverberations (yellow arrows); (E) residual blood artifact (yellow star); (F) fold over artifact (blue arrows); (G) saturation artifact (white arrow heads) \& blooming artifact (green arrow); (H) proximity artifact (red arrow head) and tangential signal drop-out (red arrow); (I) merry go round artifact (blue arrow head); (J) sunflower artifact (yellow arrow heads).

to the imaging catheter. When the imaging catheter is in the center of the lumen and parallel to the long-axis of the vessel, it results in a circular image. However, when the vessel is large in size and in vessel curvature, the catheter alignment is non-coaxial and the resulting image appears elliptical. This makes the measurements inaccurate (Figure $5 B)(2,12)$.

\section{Shadow artifact}

When light photons encounter strongly reflecting structures, the signals are reflected from the surface and do not penetrate, this results in signal drop out or shadowing behind the structure. Shadowing commonly occurs with guide wires (Figure 5B) and stent struts. In addition, air bubbles, red thrombus, blood and macrophage accumulations cause shadowing on OCT imaging. While ultrasound waves do no penetrate calcium and is an important cause of shadowing during IVUS imaging, calcium allows light photons to pass through and does not cause shadowing on OCT imaging. This allows the estimation of calcium thickness with OCT imaging $(2,12)$.

\section{Multiple reflections and Reverberations}

When light photons bump on specular surfaces, repeated reflections may happen between the transducer and the reflecting surface. This may form ghost structures at multiples of the distance between the two structures.

During OCT imaging, multiple reflections may happen within the facets of the imaging catheter that produce circular lines around the catheter (Figure 5C) or with stent struts (Figure 5D) that result in reverberations. Multiple reflection artifacts should not be mistaken for additional interfaces in the vessel or additional layer of stent struts $(2,12)$.

\section{Residual blood artifact}

Suboptimal vessel flushing causes signal rich blood swirls in the lumen that may sometimes misinterpreted as red thrombus. In addition, intense scattering of the light by red blood cells cause shadowing of the underlying structures. This makes vessel wall characterization extremely difficult (Figure 5E) $(2,12)$. 


\section{Fold over artifact}

FD-OCT has a field of view of about $10 \mathrm{~mm}$. When light is reflected from areas of vessel beyond this range aliasing occurs in the Fourier transformation that gives an appearance of the far field area of the vessel folded over in the image (Figure $5 F$ ). This artifact occurs commonly with large vessels or at areas of side branches (SBs) $(2,12)$.

\section{Saturation artifact}

Saturation artifacts are abnormally bright A-lines that appear when the high amplitude backscattered signals from specular structures like stent struts and guidewire saturate the processing limit of the detector (Figure $5 G)(2,12)$.

\section{Blooming}

Stent struts reflect light intensely and hence only the leading edge is visible on OCT images. In addition, the high signal density at the surface causes a glare or axial stretching of the stent strut reflections called blooming (Figure $5 G$ ). This makes it difficult to identify the leading edge for stent measurements $(2,12)$.

\section{Proximity artifact}

Proximity artifact refers artificial increase in the brightness of the tissues adjacent to an eccentrically positioned imaging catheter (Figure 5H).

\section{Tangential signal dropout}

Tangential signal drop out is another abnormality resulting from the eccentric catheter position. When the catheter is close to or in contact with the vessel wall, the incident light rays are parallel to the surface of the vessel wall rather than perpendicular to it. This results in artificial signal attenuation in the deeper parts of the vessel wall. With a signal rich surface and attenuated deeper tissues, this gives a false appearance of a TCFA (Figure 5H) (13).

\section{Merry-go-round effect}

The marry-go-round effect describes an apparent elongation of stent struts in the lateral direction. It results from reduced lateral resolution either from increased distance between the A-lines or larger beam spot diameter in the far field or increased scattering of the light in the lumen by red blood cells by incomplete flushing (Figure 5I) (12).

\section{Sunflower effect}

When the OCT catheter is located eccentrically in a stented vessel, the incident light beam is not perpendicular to the stent strut and instead it gets reflected only from the edge of the strut. The resultant image reconstruction orients the strut perpendicular to the catheter similar to orientation of sunflowers toward the sun. Sunflower effect gives an appearance of strut malapposition and artificially increases strut to vessel wall distance (Figure 57) (14).

\section{Clinical utility of OCT imaging}

The utility of OCT in clinical practice is (I) assessment of lesion severity, (II) evaluation of plaque vulnerability, (III) PCI optimization, and (IV) analysis of stent failure. OCT in the context of PCI optimization is described in a separate review.

\section{Assessment of lesion severity}

Various OCT parameters have been explored for the identification of flow limiting stenoses (Table 2). Although OCT MLA predicted the lesion severity in non-left main coronary artery (non-LMCA) stenosis, the cut off value varied widely from $1.6 \mathrm{~mm}^{2}$ to $2.5 \mathrm{~mm}^{2}$ between different studies and the accuracy was only modest. This limits the application of MLA for assessment of lesion severity in routine practice (15-20). The FORZA (FFR or OCT Guidance to RevasculariZe Intermediate Coronary Stenosis Using Angioplasty) study randomized subjects with intermediate lesions to OCT $v$ s. fractional flow reserve (FFR) guided decision to perform PCI. They concluded that OCT guided PCI at 13 months resulted in lower rates of the primary endpoint (composite of major adverse cardiac events or significant angina) at the expense of higher PCI rates and cost. However, significant limitations with this study limits it relevance (21). Computational processing of OCT imaging data has been used to derive an OCT based surrogate of FFR with good diagnostic accuracy and low observer variability (22). This would potentially enable combined morphological and physiological assessment of atherosclerotic lesions with the ability to use OCT to optimize PCI.

\section{Evaluation of plaque vulnerability}

Vulnerable plaque is a plaque which is prone to produce clinical events usually by plaque disruption from 


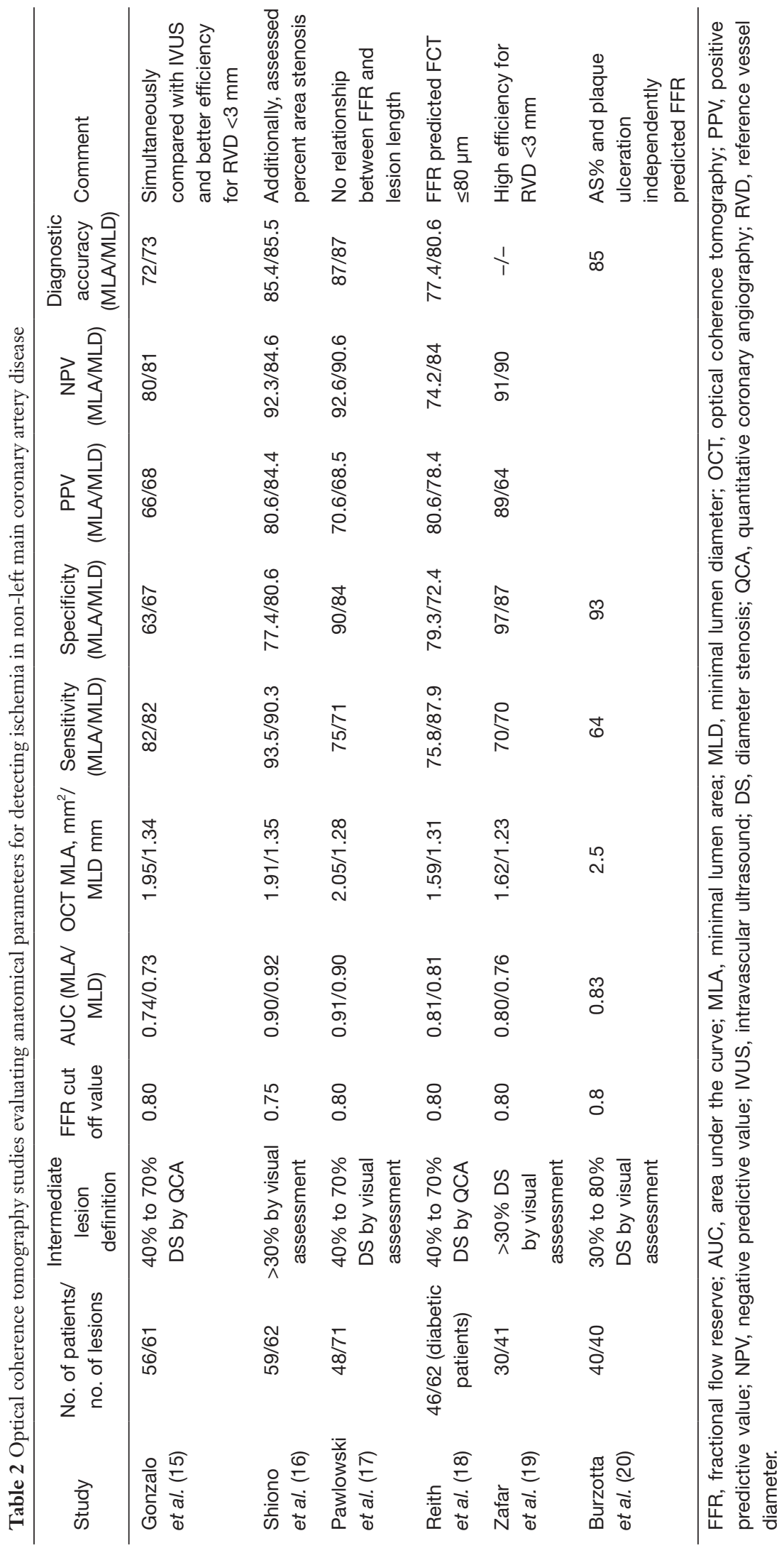


thrombosis. It is characterized by large lipid content/ necrotic core, thin fibrous cap, expansive remodeling, inflammation, spotty calcification, intraplaque hemorrhage and neoangiogenesis. Identification of such plaques may guide implementing treatment strategies which reduce the future adverse outcomes $(23,24)$. IVI modalities help not only in the identification of these unstable plaques but also in monitoring their progress with treatment. Various modalities identify different features of plaque instability. The resolution of gray scale IUVS limits it applicability in vulnerable plaque imaging. The gray scale IVUS features of vulnerability are large eccentric plaque, positive remodeling, attenuated plaque, spotty calcification and intraplaque echolucency $(24,25)$. Virtual histology defined TCFA is an important predictor of adverse events. In the PROSPECT (Providing Regional Observations to Study Predictors of Events in the Coronary tree) study, TCFA along with a plaque burden of $\geq 70 \%$ and a lumen area $\leq 4 \mathrm{~mm}^{2}$ were associated with clinical events in non-culprit lesions during follow up (26). OCT with its resolution capabilities identify the features of vulnerability with high accuracy. Lesions with OCT defined TCFA, lipid rich plaque, microchannels, spotty calcification, macrophages and intraluminal thrombi (Figure 3) have been shown to progress at 7-month follow up (27). However, the natural history of vulnerable plaque is too complex, with most of the TCFA stabilize and few thick cap fibroatheroma transform into TCFA. Thus, the treatment of atherosclerosis still remains at the patient level rather than at the plaque level (28).

\section{Utility in ACS}

ACS represents a high-risk subset of patients undergoing PCI. Although PCI in ACS patients improves clinical outcomes, it is also associated with inferior procedural outcomes from distal embolization, no-reflow and resultant periprocedural MI and higher incidence of drug eluting stent failure (29). The prominent roles OCT imaging in ACS patients undergoing revascularization are: (I) identification of the culprit lesion, (II) characterization of the underlying pathology, (III) prediction of no-reflow and (IV) stent optimization. The following discussion focuses on the first three aspects and stent optimization is described elsewhere in this series.

\section{Identification of culprit lesion}

In about $30 \%$ of patients presenting with ACS no clear culprit lesion is identified [MI with non-obstructed coronary arteries (MINOCA)] while in approximately 10\% there are multiple culprits. In patients with MINOCA and no angiographically identifiable culprit lesion, OCT imaging could be an invaluable tool to detect atherosclerotic plaque disruption or nonatherosclerotic arterial pathology or to exclude a coronary arterial cause for the presentation. OCT has greatly increased the awareness and diagnosis of nonatherosclerotic ACS events such as spontaneous coronary artery dissection (SCAD). In the recent SOFT-MI study coronary plaque disruption and thrombus were present in $24 \%$ and $18 \%$ of MINOCA patients respectively (30). In patients with multiple PRs, the culprit lesion is more often associated with larger plaque burden, smaller lumen area and thrombus (31).

\section{Identification of ACS lesion morphology to guide management strategy}

ACS primarily results from disruption of atherosclerotic plaques and less often from the nonatherosclerotic mechanisms such as spontaneous coronary dissection, coronary spasm and thromboembolism. The predominant mechanisms of ACS from atherosclerotic plaques are PR, $\mathrm{PE}$ and eruptive calcified nodule (ECN). OCT, can identify all of these pathological mechanisms of ACS and with a high degree of accuracy in vivo and is now increasing recognised as a potential tool not just for the study of these patients but to guide tailored therapy (32).

$\mathrm{PR}$ is the most prevalent and frequently studied mechanism of ACS and accounts for up to two-thirds of all ACS cases. Most of therapeutic strategies for management of ACS have been developed based on the pathophysiology of PR. PR results from disruption of TCFA that exposes the underlying highly thrombogenic necrotic core directly to the circulating blood promoting thrombosis (Figure 3I). It is associated with gross distortion of the vessel architecture with positive remodelling, large necrotic core, fibrous cap disruption, intraplaque cavity, large thrombus burden and a small residual lumen area. In addition, the necrotic core remnants may act as a persistent stimulus for thrombosis and re-occlusion following successful thrombolysis. Stent implantation seals the plaque and improves the flow area. Thus, drug-eluting stent (DES) implantation along with antithrombotic therapy has become the standard of care for the management of ACS $(33,34)$. Whether PR in plaques with a large residual lumen can be managed with medical therapy without the need for stenting remains to be evaluated. 

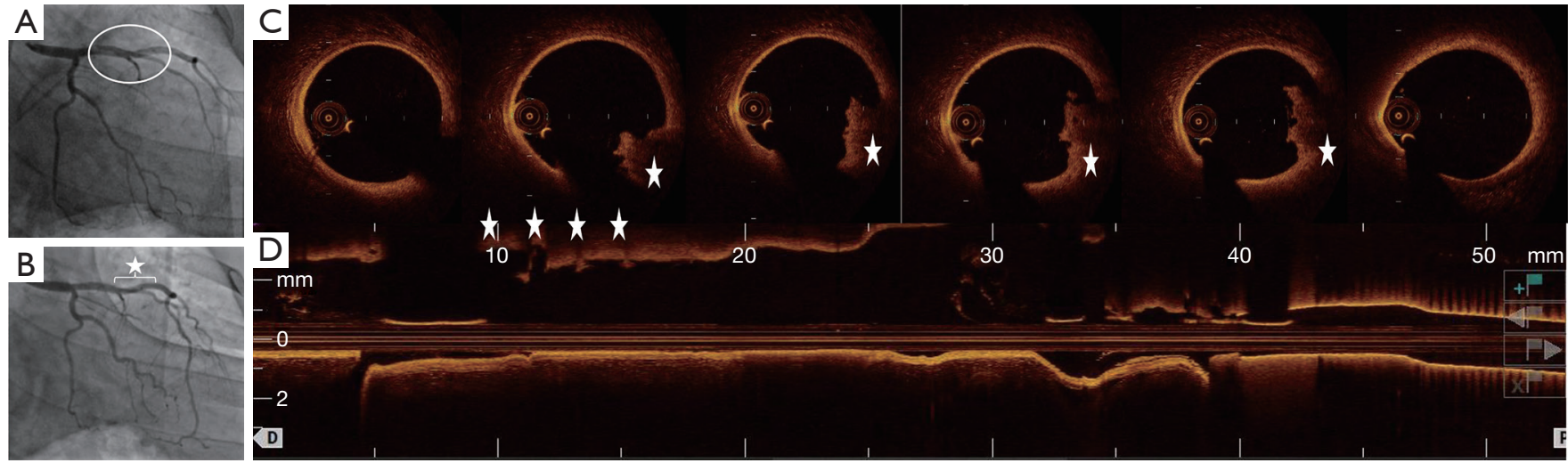

Figure 6 Definite plaque erosion. A 40-year-old gentleman presented to a peripheral hospital with acute anterior wall myocardial infarction and thrombolysed with tenecteplase. He underwent coronary angiogram (CAG) as part of pharmacoinvasive percutaneous coronary intervention (PCI). CAG showed proximal left anterior descending coronary artery lesion with large thrombus burden (white circle) and thrombolysis in myocardial infarction (TIMI) III flow (A). PCI was deferred and he was treated with low molecular weight heparin for 5 days. His repeat CAG showed good thrombus resolution (B, star). Optical coherence tomography (OCT) imaging showed small mixed thrombus attached to the intimal surface without lumen compromise (C,D, white stars). He was treated with dual antiplatelet therapy.

$\mathrm{PE}$ is responsible for a quarter of ACS and results from disruption of the endothelial layer covering the plaque. In contrast to PR, the vessel architecture is mostly preserved with a smaller plaque burden, intact fibrous cap, smaller thrombus volume and a larger residual lumen area. Once the thrombus is treated, the thrombogenicity of the plaque is limited to the exposed subendothelial surface which can be managed with antithrombotic therapy alone without the need for stent implantation (Figure 6). This may eliminate the long-term risk of stent thrombosis, in-stent restenosis and dual antiplatelet therapy. There is now a great degree of enthusiasm to pursue this management strategy (35-38).

Prati $e$ al. treated 31 patients with OCT identified PE either with thrombectomy only (no stent group, $n=12$ ) or thrombectomy followed by stenting (stent group, $\mathrm{n}=19$ ). All patients were kept on DAPT (aspirin + clopidogrel or prasugrel). The median follow-up was 753 days. A patient in the stent group required repeat revascularization, and there was no death, MI, or heart failure admission in either group (39).

This strategy was further evaluated in the prospective EROSION (Effective anti-thrombotic therapy without stenting: intravascular OCT-based management in PE) study. Of the 405 ACS patients enrolled in the study, PE was observed in $103(25.4 \%)$ patients and 60 of them met the additional inclusion criteria of $<70 \%$ diameter stenosis. Thrombus aspiration and GP IIb/IIIa inhibitor administration were left to operator discretion. All patients were treated with aspirin and ticagrelor and 3 days of heparin. Fifty-five patients completed the 1-month OCT follow-up. The primary end point of $>50 \%$ reduction in thrombus volume on OCT imaging at month was met in $47(78.3 \%)$ patients and complete thrombus resolution was observed in 22 patients. The mean thrombus volume reduced from 3.7 to $0.2 \mathrm{~mm}^{3}$ and minimal flow area improved from 1.7 to $2.1 \mathrm{~mm}^{2}$ (38). Forty-nine patients underwent repeat OCT imaging at 1-year that showed further reduction in thrombus volume from 0.3 to $0.1 \mathrm{~mm}^{3}$ $(\mathrm{P}=0.001)$. Overall, 4 (7.6\%) patients developed major adverse cardio-vascular events (3 revascularizations and 1 major bleed) which is on par with standard of care DES and DAPT (40). Although the ani-thrombotic alone strategy for non-flow limiting PE appears promising, this needs to be evaluated in large scale prospective randomized studies (41).

ECN is the third common cause of ACS with a prevalence of $2-8 \%$ (7). It occurs more often in elderly patients and those with chronic kidney disease on haemodialysis. It is proposed to evolve from fragmentation of superficial calcium sheets due to mechanical stress and commonly observed in the mid right coronary artery. On OCT imaging, it appears as a protruding calcific mass with irregular surface and dorsal shadowing. PCI in patients with $\mathrm{ECN}$ is challenging, as it occurs in the background of extensive superficial calcification and vessel tortuosity. Lesions with ECN are associated with small thrombus burden and small or no necrotic core and often 


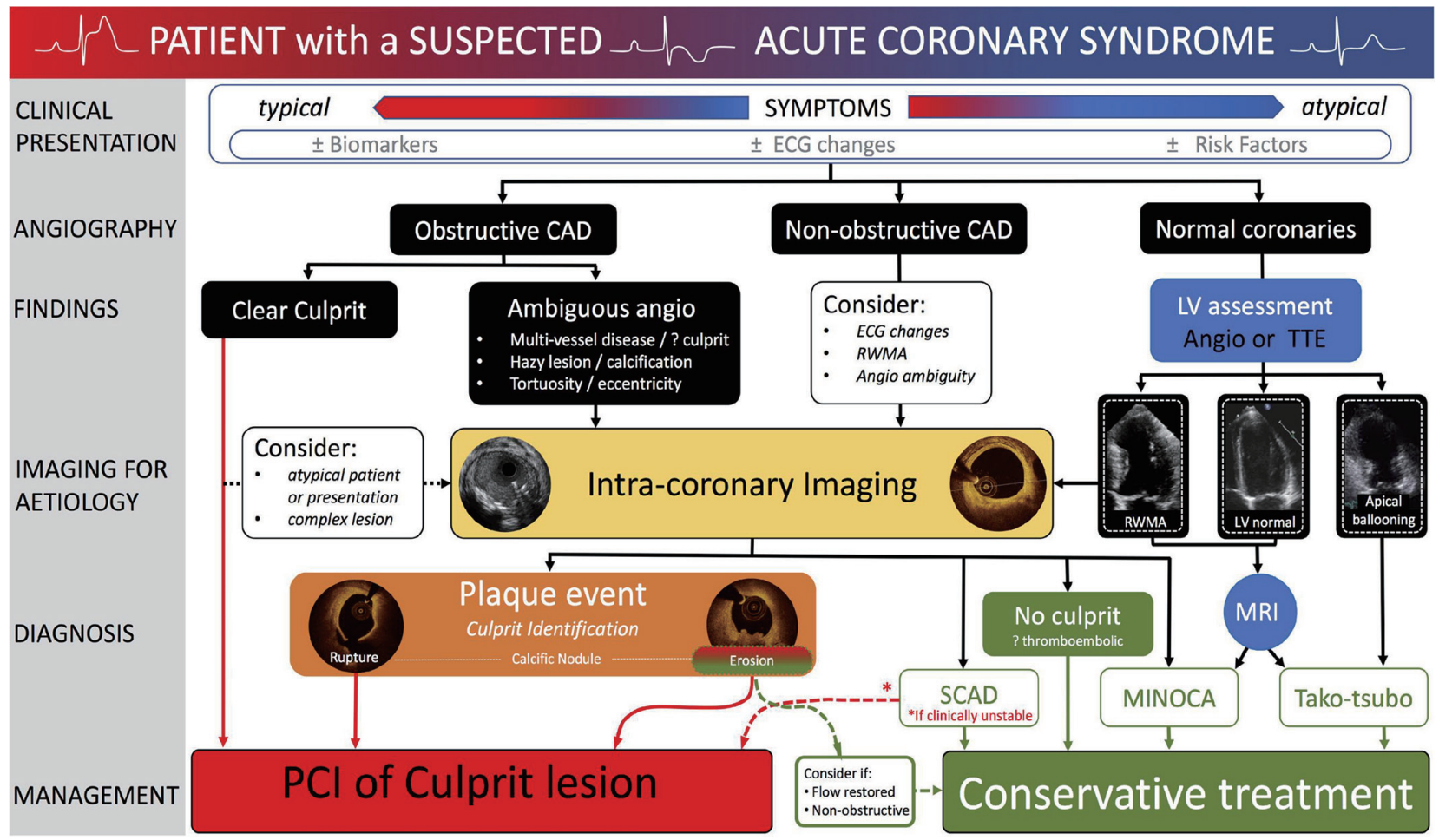

Figure 7 Treatment algorithm to guide the use of intravascular imaging in patients presenting with acute coronary syndromes. CAD, coronary artery disease; ECG, electrocardiogram; LV, left ventricle; TTE, transthoracic echocardiogram; SCAD, spontaneous coronary artery dissection; PCI, percutaneous coronary intervention; RWMA, regional wall motion abnormality; MRI, magnetic resonance imaging; MINOCA, myocardial infarction with non-obstructed coronary arteries. Johnson et al. (32). Reprinted by permission of Oxford University Press on behalf of the European Society of Cardiology.

need aggressive preparation with balloon dilation and atherectomy. OCT imaging helps in selecting the debulking strategy, assessing the response to debulking and finally stent optimization $(34,42)$ (Figure $3 L$ ).

An IVI based algorithm has been proposed for the assessment and management of patients with ACS (Figure 7).

\section{Spontaneous coronary dissection}

SCAD is a non-atherosclerotic, non-iatrogenic, and nontraumatic separation of epicardial coronary artery walls. It accounts for $1-4 \%$ of all ACS cases and is more common in young and middle-aged females $(43,44)$. Intramural hematomata (IMH), the characteristic of SCAD, results either from intimal tear with ensuing entry of blood into the false lumen or from rupture of the vaso-vasorum within the vessel wall.

Although CAG remains the initial modality for evaluation of patients with suspected SCAD, its inability to visualize the vessel wall limits its utility in this condition. Hence, guidelines recommend adjunctive IVI, in case of non-diagnostic CAG and there is a high index of clinical suspicion of SCAD and, particularly when a subsequent intervention is planned (44). Diagnosis of SCAD on OCT imaging is based on the documentation of the intimomedial membrane with true and false lumens (double lumen appearance) or IMH. In addition, it also provides useful information on intimal rupture, thrombus, longitudinal and circumferential extent of disease, involvement of SBs and lumen compromise (Figure 8) (45).

A conservative approach is the standard of care in patients with SCAD as most of the dissections heal spontaneously. Revascularization is recommended only in the presence of high-risk features such as ongoing ischemia, hemodynamic or electrical instability and severe LMCA involvement. PCI in patients with SCAD is associated with a higher incidence of complications and lower procedural success rate. During 

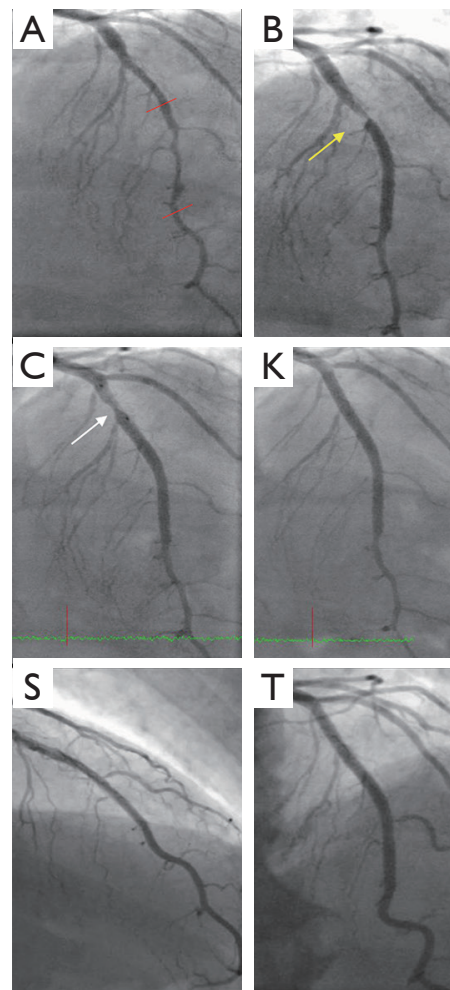
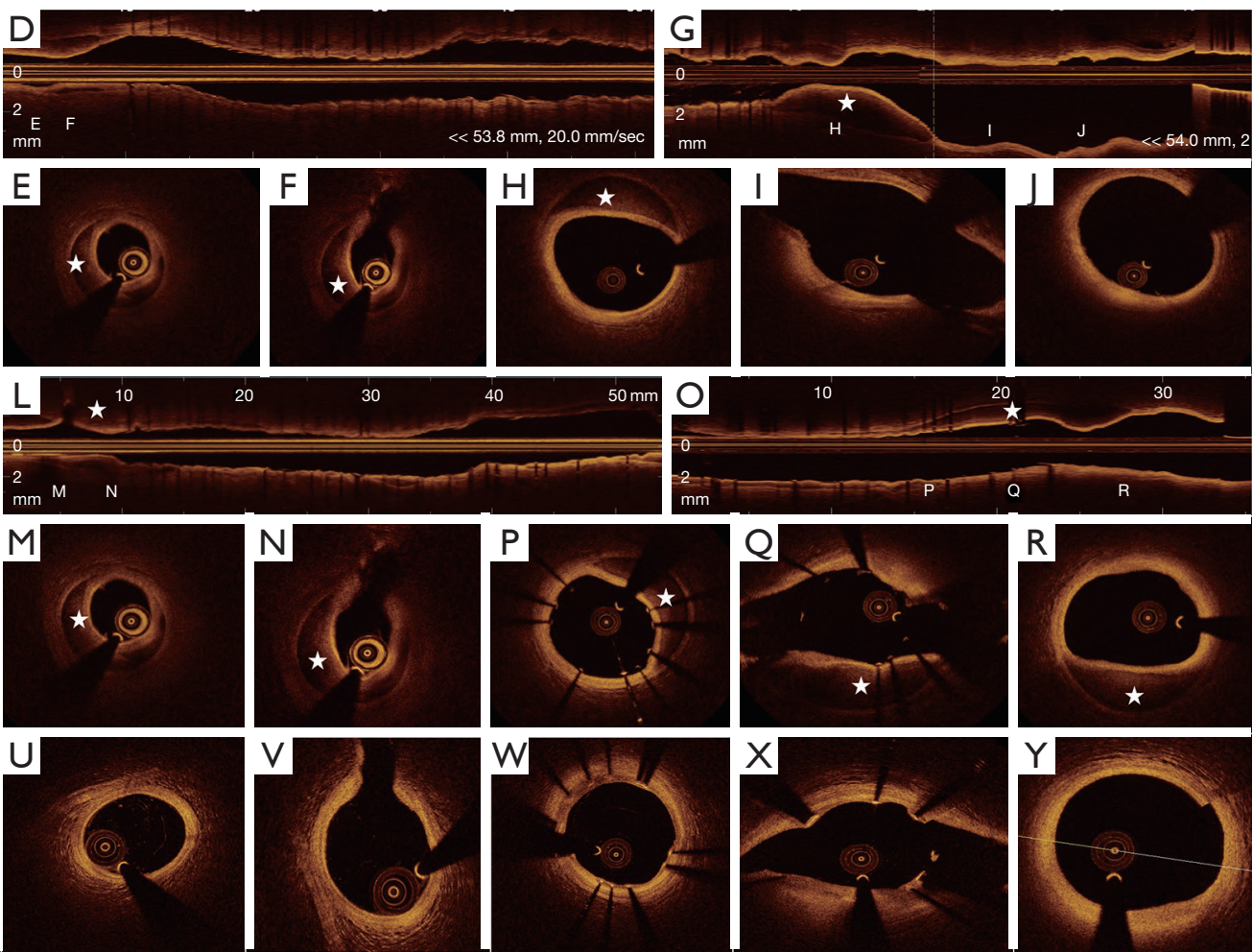

Figure 8 Spontaneous coronary dissection. A 52-year-old female presented with non-ST-segment elevation myocardial infarction. Her coronary angiogram (A) showed atherosclerosis like lesion in the distal left anterior descending coronary artery (LAD, lesion is marked with red lines). She underwent drug-eluting stent (DES) implantation for the same. Post-stenting CAG showed hazy lesion suggestive of dissection/hematoma at the proximal edge (B, yellow arrow). Additional DES was implanted proximally. A similar looking lesion appeared at the proximal edge $(\mathrm{C}$, white arrow). At this stage, OCT imaging was performed which showed intramural hematoma at both distal [(D) long view; (E,F) cross sectional views showing distal edge hematoma, stars)] and proximal edges $[(\mathrm{G})$ long view; (H) cross sectional view showing distal edge hematoma, star; (I) LAD/diagonal/septal trifurcation and proximal vessel free of hematoma; (J) proximal LAD]. The proximal hematoma was covered with a third DES (K). Final OCT showed distal edge hematoma (stars) which was non-progressive [(L) long view; $(\mathrm{M}, \mathrm{N})$ cross sectional views] and hematoma extension proximally beyond the diagonal branch (stars) [(O) long view; (P,Q,R) cross sectional views]. (S,T) Follow-up CAG at 6 months showing normal appearing stent edges. (U,V,W,X,Y) Corresponding OCT cross-sectional views showing complete healing of the hematoma. Stars indicate intramural hematoma. CAG, coronary angiography; OCT, optical coherence tomography.

PCI, OCT confirms position of the guide wire in the true lumen as inadvertent false lumen stenting may be associated with potentially serious consequences. It helps in stent sizing as stent oversizing may result in extension of hematoma or vessel rupture. In addition, it also provides true longitudinal extent of IMH that ensures optimal lesion coverage. Further, it aids in planning stenting strategy based on the presence or absence of entry and exit tears. In case of entry only tear, distal to proximal stent implantation and in exit only tear, proximal to distal stent implantation may prevent extension of the hematoma. In the absence of intimal tears, sealing either ends of the hematoma before tackling the middle part may avoid hematoma propagation (46). Otherwise, cutting balloon dilatation may also be used to decompress the hematoma (47). In addition, stent oversizing and aggressive post dilatation should be avoided $(46,48)$.

\section{Coronary vasospasm}

Coronary vasospasm is an important cause of ACS and should be considered in those presenting with ACS and non-obstructive CAD. Its prevalence varies widely in different studies (49). The key role of OCT imaging in these patients is to eliminate other causes of ACS such as PE 
and SCAD and identification of angiographically invisible thrombus. The characteristic OCT features of coronary spasm are thickened media with intimal bumps (smooth intimal projections) at rest and intima gathering (multiple intimal kinks) during spasm. The intimal changes typically disappear with intracoronary injection of nitroglycerine. In addition, the spasmodic sites more often show features of erosion such as lumen irregularity and thrombus (50-52).

\section{IVI-guided intervention to prevent no-reflow}

No-reflow during PCI remains an important predictor of poor acute and long-term outcomes. In ACS patients, distal embolization is one of the major predictors of noreflow. In a small OCT study of 25 patients, the different predictors of filter no reflow [FNR, thrombolysis in myocardial infarction (TIMI) flow grade 0 after balloon dilatation] were stent length, lipid pool length, lipid pool + thrombus index and lipid pool + thrombus length. In multivariate analysis, the only independent predictor of FNF was lipid pool + thrombus length. The optimal cutoff length that predicted FNR was $13.1 \mathrm{~mm}$. Hence, use of embolic protection devices in those with OCT identified large atherothrombotic burden may reduce the incidence of no-reflow (53).

\section{Bifurcation lesions}

Coronary bifurcation lesions (CBL) account for $15-20 \%$ of all PCIs and are associated with inferior procedure success rates and higher incidence of adverse outcomes compared to non-bifurcation PCIs $(54,55)$. With its anatomical and procedural complexity, angiography alone is insufficient for procedure guidance. OCT with its high resolution and its capacity to provide three-dimensional imaging (3D bifurcation mode), stent rendering and apposition indicator functions is aptly suited for image guidance during bifurcation stenting. The three important roles of OCT imaging during bifurcation stenting are (I) prediction of SB compromise, (II) assessment of the jailed SB ostium and confirmation of optimal SB wire re-crossing, (III) stent size selection and stent optimization.

Carina shift is the most important mechanism of SB compromise following provisional stenting strategy. OCT parameters associated with carina shift after implanting main vessel stent are (I) narrow carina tip angle $\left(<50^{\circ}\right)$, (II) short branch point to carina tip length $(<1.7 \mathrm{~mm})$ and (III) plaque distribution opposite to the SB. Following main branch (MB) stenting, SB ostium is jailed with stent struts that acts as nidus for thrombus formation. In addition, neointimal coverage of the jailed struts results in SB stenosis and also prevent future access to the SB. Although routine kissing balloon dilatation (KBD) was shown to reduce the incidence of $\mathrm{SB}$ ostial stenosis, it increased the incidence of $\mathrm{MB}$ restenosis and repeat revascularization. 3D OCT imaging identifies two different morphological types of jailed SB ostia, connecting to the carina (distal link attached to the carina) and free carina type (no link at the carina) and in addition confirms distal cell crossing of the guidewire. In the free carina type, distal cell crossing is associated with complete unjailing of the SB ostium (Figure 9). Proximal cell crossing in the free carina type and crossing at either location in the connecting to carina type may result in stent distortion and malapposition at the SB ostia. Similarly, during 2-stent strategy, OCT confirms mid to distal crossing before balloon dilatation. Further, OCT imaging also reveals inadvertent abluminal rewiring. Bifurcation stenting, particularly the 2 -stent techniques are associated with a high incidence of underexpansion and malapposition. OCT imaging with its stent rendering, apposition and expansion indicator functions facilitates identification of these abnormalities and hence appropriate corrective actions can be taken to avoid future adverse events (56-61). Whether the use routine use of OCT to guide bifurcation PCI is associated with better outcomes is being tested in ongoing trials (62).

\section{Calcified lesions}

Coronary artery calcification (CAC) is a predictor of difficult lesion preparation and device delivery during PCI and also associated with an increased incidence of underexpansion, malapposition and periprocedural MI $(63,64)$. Although both IVUS and OCT identify calcium with similar efficiency, OCT imaging provides additional information on calcium plate thickness, a valuable parameter for selecting lesion the lesion modification strategy (65). The various roles of OCT imaging during calcified lesion PCI (CL-PCI) are: (I) prediction of the need for atherectomy, (II) confirmation of adequacy of lesion preparation, (III) verification of optimal stent expansion. Fujino et al., developed an OCT based scoring system by assigning 2 points to calcium arc of $>180^{\circ}$ and one point each to thickness $>0.5 \mathrm{~mm}$ and length $>5 \mathrm{~mm}$ and assessed stent expansion with balloon only pre-treatment. A score of 3 or less was associated with optimal stent expansion 
and a score of 4 predicted stent underexpansion. After atherectomy, OCT imaging may be used to assess the residual calcium plate thickness. A residual calcium plate thickness at its thinnest part $<500 \mu$ predicts calcium plate fracture with balloon dilatation. The more the number of fractures seen in the calcified plate, the better is stent expansion. In addition, post stenting OCT confirms optimal stent expansion (66-68) (Figure 10).

\section{Cardiac allograft vasculopathy (CAV)}

CAV is the major cause of late allograft failure. It is observed in about half of the patients at 10 years (69). Early CAV takes the form of diffuse intimal thickening and atherosclerotic changes develop in the later stages. CAG is insensitive to detect the early changes of CAV which is often diffuse and non-flow limiting (70). OCT, with its ability to detect early intimal changes of CAV, has become the modality of choice to diagnose and monitor the progression of CAV. Further, OCT detects atherosclerotic changes more often than IVUS. Hou et al. assessed left anterior descending coronary artery in seven long-term transplant survivors with both OCT and IVUS imaging (71). Compared to IVUS, OCT imaging more frequently identified intimal hyperplasia (intimal thickness $>100 \mu$ ) and lipid rich plaques with thin fibrous caps. In another study by Cassar et al. the prevalence of both atherosclerotic changes (eccentric plaques, lipid pools, macrophages, microchannels, and calcification) and complicated lesions (PR, intimal laceration, and intraluminal thrombus) increased with the duration of transplantation (72). In a recent OCT study, the extent of layered fibrous plaques (homogeneous, signalrich tissue in a layered pattern) and intimal bright spots were predictive of nonfatal progression of CAV $(73,74)$ (Figure 11).

\section{Stent failure}

IVI is vital in identifying the mechanisms of in stent restenosis (ISR) or stent thrombosis. This then helps guide appropriate treatment to mitigate the risk of recurrent stent failure. OCT is particularly advantageous in this situation and should be strongly considered in cases of ISR and if available is mandatory in stent thrombosis (75).

\section{OCT imaging to identify causes of stent failure}

At the coronary arterial level, stent failure occurs due to a stent "mechanical" abnormality (suboptimal stent deployment and stent structural abnormalities) and/or abnormal neointimal healing (76).

OCT with its high resolution clearly reveals the characteristics of suboptimal stent deployment (underexpansion, malapposition, stent edge dissection or hematoma, geographic miss, tissue prolapse) and stent structural abnormalities (stent fracture, stent gap, longitudinal deformation) (77-79).

A normal neointimal response to stenting results in the development of a thin homogeneous layer of neointima that is uniform around the circumference of the stent with complete coverage of all stent struts. Stent failure can occur when neointimal growth is reduced, with incomplete strut coverage being a nidus for stent thrombosis. Alternatively, neointimal tissue growth can be proliferative and abnormal with several different OCT patterns recognised. Neointimal proliferation has been categorised into four different OCT patterns: homogeneous (high backscattering and low attenuating tissue), layered (high backscattering abluminal layer and a low backscattering abluminal layer), heterogeneous (tissue with non-uniform optical properties with areas of high and low backscattering throughout) and neoatherosclerosis (atherosclerotic changes in the neointimal tissue) (80). More recently up to seven different OCT patterns have been suggested $(81,82)$. The different neointimal patterns represent dissimilar tissue composition and the response to treatment (plain balloon dilatation vs. drug eluting balloon $v s$. drug eluting stent) also may vary (83). OCT imaging, by portraying these pathophysiological mechanisms in vivo, enables individualized treatment of stent failure.

\section{Neoatherosclerosis}

Neoatherosclerosis refers to the development of typical atherosclerotic changes within the neointimal tissue. It is being increasingly recognized as an important cause of both ISR and very late stent thrombosis with both bare metal stent (BMS) and DES. The endothelium covering the neointimal tissue is dysfunctional and promotes transmigration of macrophages and accumulation of lipoproteins resulting in the atherosclerotic changes seen in stent Neoatherosclerosis. This result either in a progressive increase in the atherosclerotic tissue volume with resultant ISR or plaque disruption with stent thrombosis $(84,85)$. With OCT, it is currently possible to diagnose 

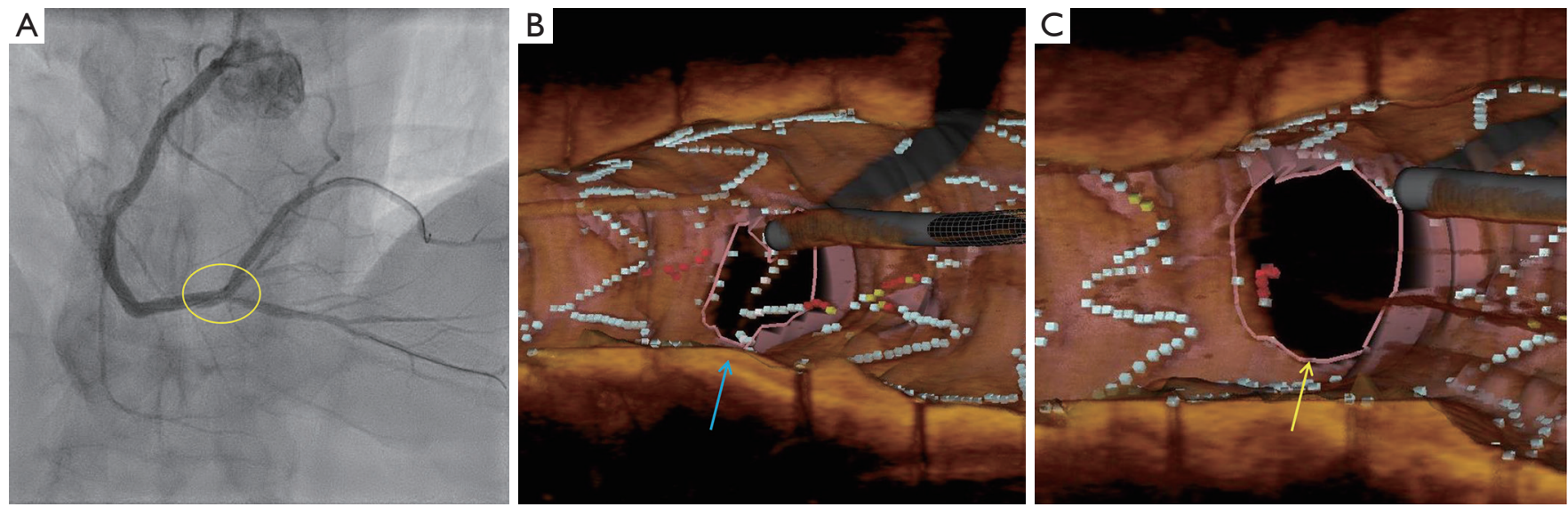

Figure 9 OCT-guided provisional stenting to distal RCA bifurcation. (A) Coronary angiogram showing distal RCA bifurcation post provisional stenting into postero-lateral branch (yellow circle). (B) 3D bifurcation view showing link free type side branch ostial strut morphology and distal cell wire crossing (blue arrow). (C) 3D bifurcation view post kissing balloon dilatation showing the side branch ostium free of stent struts (yellow arrow). OCT, optical coherence tomography; RCA, right coronary artery.
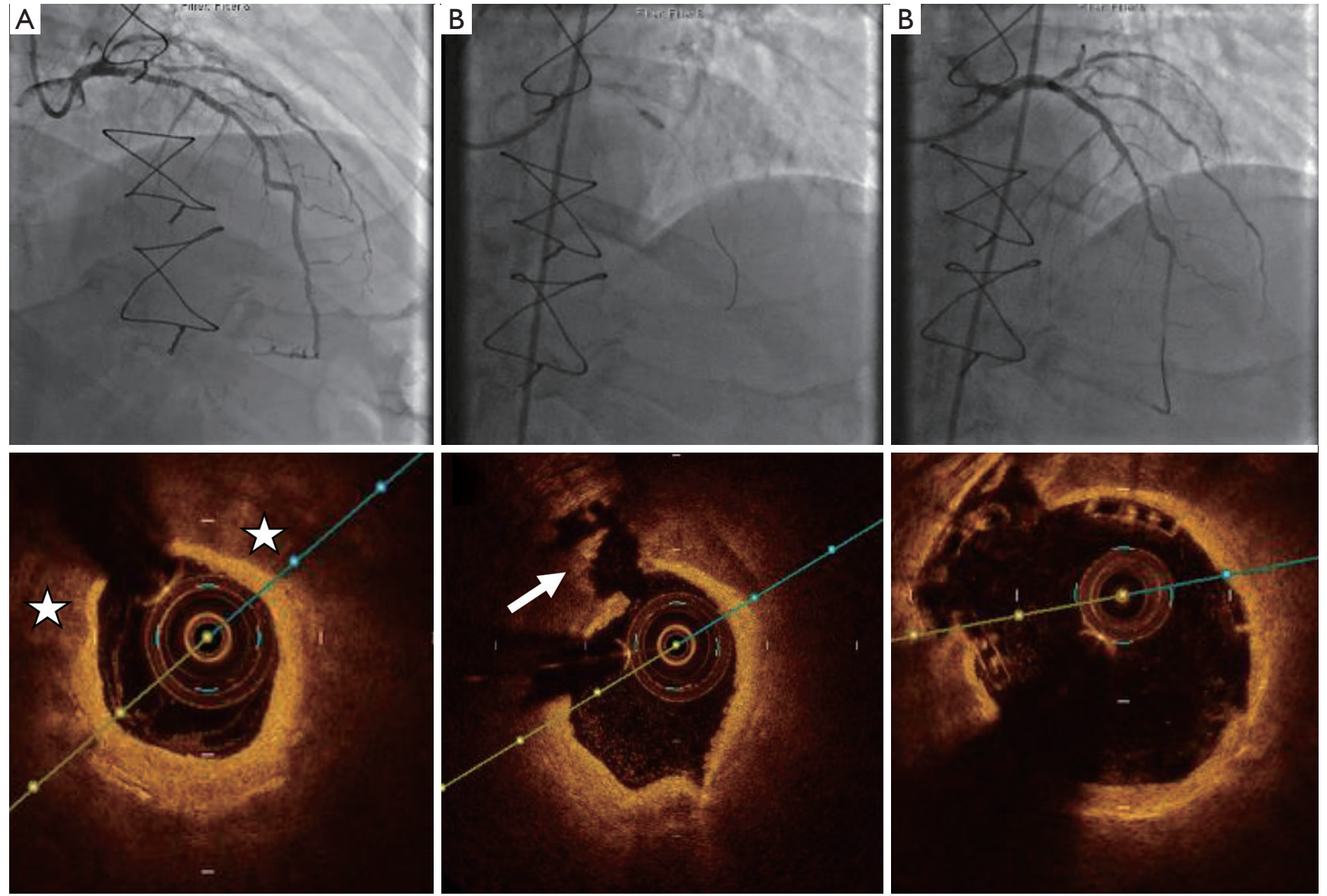

Figure 10 OCT-guided bioresorbable scaffold implantation in a severely calcified lesion (stars). (A) Baseline angiographic and OCT images. OCT image showing $180^{\circ}$ lesion calcification. (B) Post cutting balloon dilatation OCT image showing crack (arrow) at 11 o'clock position. (C) Final images showing optimally expanded scaffold. 

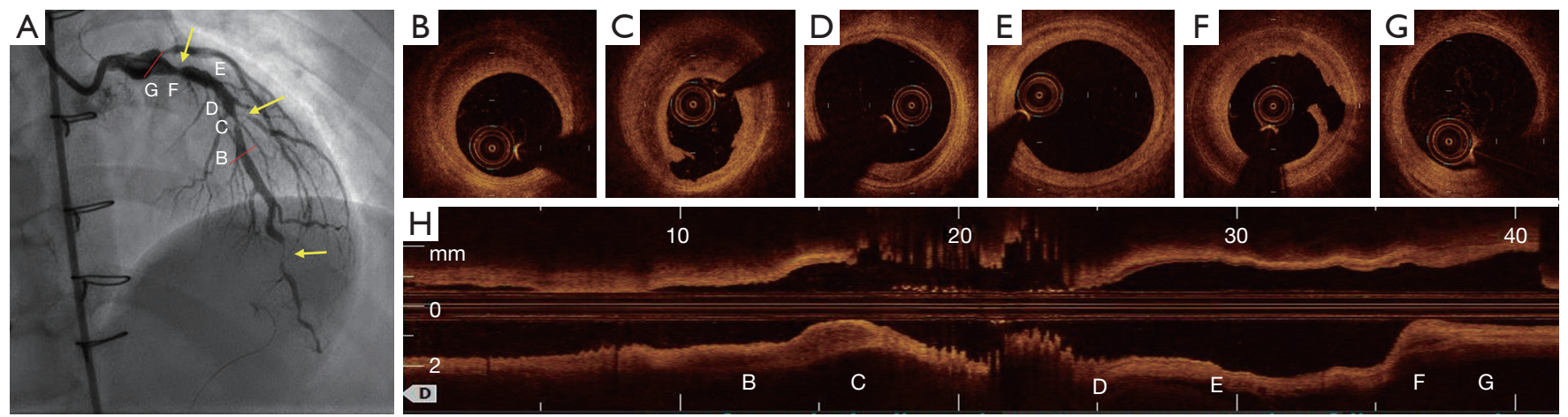

10

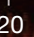

Figure 11 Transplant vasculopathy in a patient 3 years post-transplantation. (A) CAG showing three focal stenoses in left anterior descending coronary artery (arrows). (B,C,D,E,F,G) OCT cross-sectional images of mid- to ostio-proximal segment. OCT frames (B,C,D) and (F) show layered fibrous plaque (the dark appearance of the plaque results from immature fibrous tissue). (E) and (G) show intimal thickening. Balloon dilatation induced dissection is shown at 6 o'clock position in (C) and 3 o'clock position in (F). Longitudinal OCT view is shown in $(\mathrm{H})$. Red lines on CAG indicate the proximal and distal limits of OCT imaging. OCT, optical coherence tomography; CAG, coronary angiography.

Table 3 OCT abnormalities in patients with in-stent restenosis and stent thrombosis

\begin{tabular}{|c|c|}
\hline Stent failure mode & Abnormalities \\
\hline Early & Stent under-expansion; geographic miss; stent gap; longitudinal deformation; neointimal hyperplasia \\
\hline Late & Neoatherosclerosis; stent fracture \\
\hline \multicolumn{2}{|l|}{ Stent thrombosis } \\
\hline Late & $\begin{array}{l}\text { Uncovered stent struts; malapposition; underexpansion; edge pathology (geographic miss, dissection/ } \\
\text { hematoma); in-stent restenosis; ruptured neoatherosclerosis; coronary evaginations }\end{array}$ \\
\hline Very late & $\begin{array}{l}\text { Uncovered stent struts; malapposition; underexpansion; edge pathology (geographic miss, dissection/ } \\
\text { hematoma); ins-tent restenosis; ruptured neoatherosclerosis; coronary evaginations }\end{array}$ \\
\hline
\end{tabular}

OCT, optical coherence tomography.

neoatherosclerosis in vivo. It is characterized by lipid laden intima, calcification, intimal rupture and luminal thrombus (Figure 12). Although it was perceived that stent restenosis is a benign process presenting with chronic stable angina and stent thrombosis as acute presentation with dramatic outcomes, a significant number of patients with late ISR present with ACS and neoatherosclerosis with rupture serves as the common denominator between the two forms of late stent failure.

Table 3 summarizes the various OCT abnormalities observed in patients with stent failure and Figures 13,14 summarizes the targeted treatment of ISR and stent thrombosis based on the underlying pathology.

\section{Discontinuation of dual antiplatelet therapy}

Premature discontinuation of DAPT is sometimes required for clinical reasons. This may be associated with an increased incidence of stent thrombosis. OCT clearly delineates various features of suboptimal deployment, such as underexpansion, malapposition and edge abnormalities and in addition, its high resolution enables assessment of neointimal coverage. This may provide an opportunity to risk stratify patients who require temporary discontinuation 

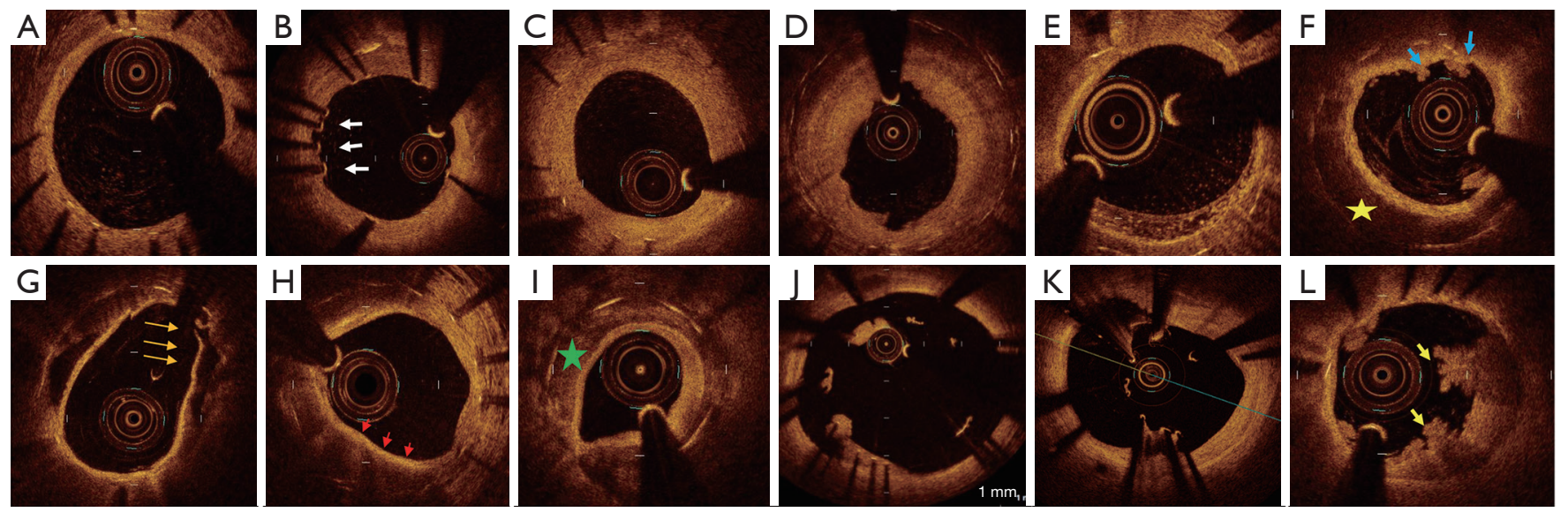

Figure 12 OCT images of strut coverage (A,B) and stent failure (C,D,E,F,G,H,I,J,K,L). (A) Struts coverage with thin homogenous neointima; (B) uncovered stent struts (white arrows); (C,D,E) abnormal neointimal growth with homogeneous pattern (C), layered pattern (D), heterogeneous pattern (E); (F,G,H,I) neoatherosclerosis with lipid rich plaque (yellow star) and white thrombus (blue arrows) (F), plaque rupture (orange arrows) (G), macrophages (red arrows) (H) and calcification (green star) (I); (J,K,L) stent thrombosis with malapposed stent struts $(\mathrm{J})$, late acquired malapposition $(\mathrm{K})$ and persistently uncovered stent struts (yellow arrows indicate white thrombus) (L).

\section{of DAPT.}

Iliescu et al., evaluated 40 cancer patients who required transient discontinuation of DAPT with OCT imaging (86). Patients were divided into two groups based on the following features: underexpansion, malapposition, uncovered stent struts, ISR or intraluminal mass. Twentyseven patients $(68 \%)$ without these features were considered low risk and allowed to discontinue DAPT. The remaining 13 patients with one or more of these features were considered high risk and underwent further intervention. At 12-month follow-up, there were no cardiac events in the low risk group and one MI occurred in the high risk. Fourteen patients died of cancer progression during this period. Although this approach is intuitive, it needs to be evaluated in a large population study.

\section{Minimal or zero contrast angioplasty}

Contrast induced nephropathy (CIN) is a rare but potentially serious complication following angiographic procedures. Although numerous strategies have been tested, none of them except for a reduction in the volume of contrast administered have been shown to reduce the incidence of CIN. Hence, a significant proportion of CAD patients with renal dysfunction are denied diagnostic and therapeutic angiographic procedures. IVI guidance during invasive procedures, reduce the need for multiple contrast injections that in turn reduce the volume of contrast administered. This led to the development of IVI guided minimum or zero contrast PCI (87).

While this strategy has been primarily used IVUS, recently, zero contrast PCI has also been reported with OCT guidance using low molecular weight dextran. The additional advantage with OCT guided zero contrast PCI, is the feasibility of co-registration of fluoroscopic images with OCT longitudinal and cross-sectional images $(88,89)$.

\section{Conclusions}

Since the introduction of commercial OCT systems almost 2 decades ago, OCT with its almost microscopic demonstration of in vivo coronary arterial anatomy has provided unprecedented insights into atherosclerotic coronary disease and the response of the coronary artery to PCI. With the development of rapid scanning systems, continued development of the technology and analysis software it has also become an invaluable tool in the catheterization laboratory for the assessment and management of specific patient subsets based on clinical need. Whether routine use of OCT to guide and optimize PCI will become standard of care remains to be proven. 


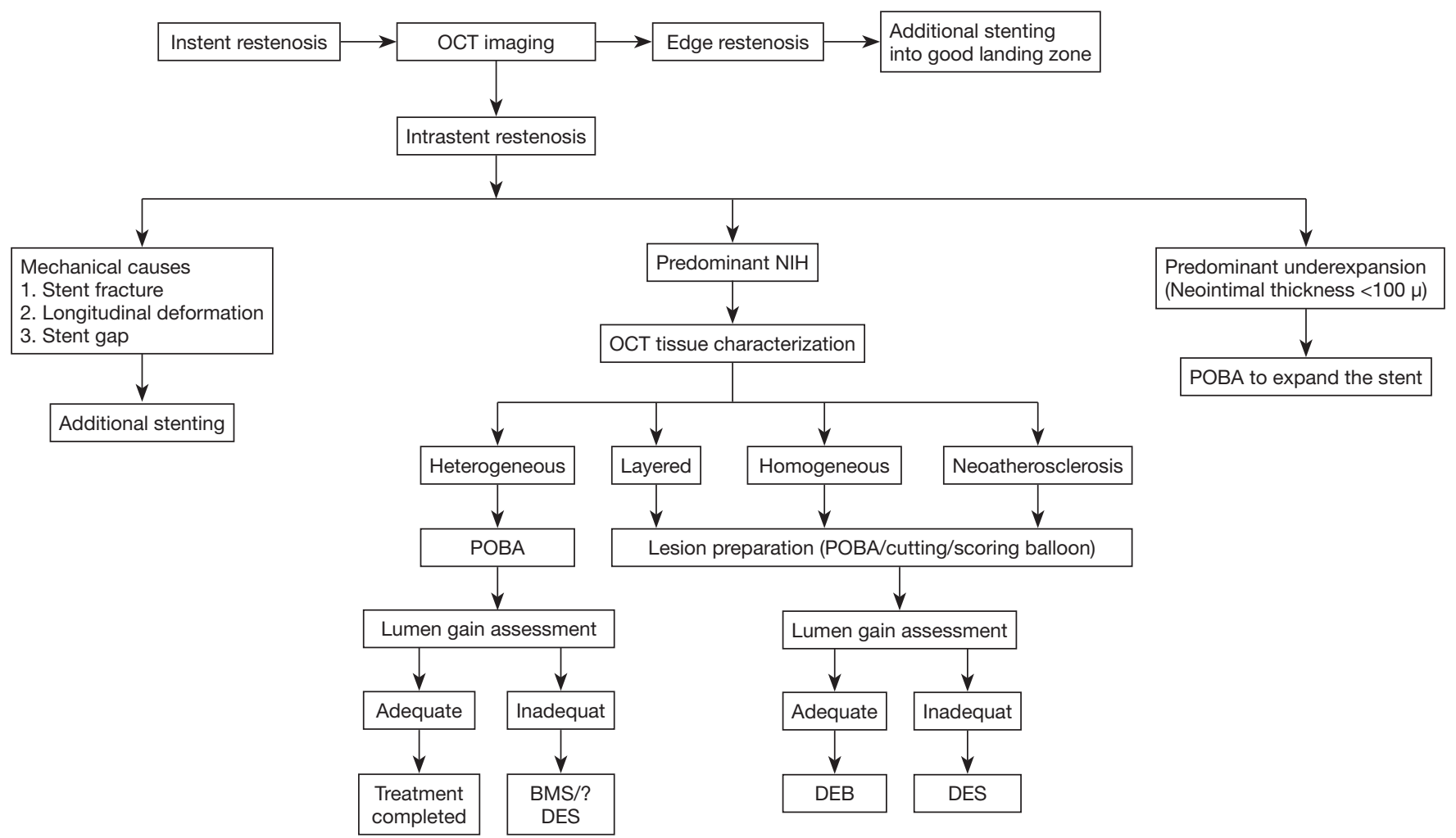

Figure 13 OCT-guided treatment of in-stent restenosis. OCT, optical coherence tomography; NIH, neointimal hyperplasia; POBA, plain ordinary balloon angioplasty; BMS, bare metal stent; DES, drug-eluting stent; DEB, drug-eluting balloon.

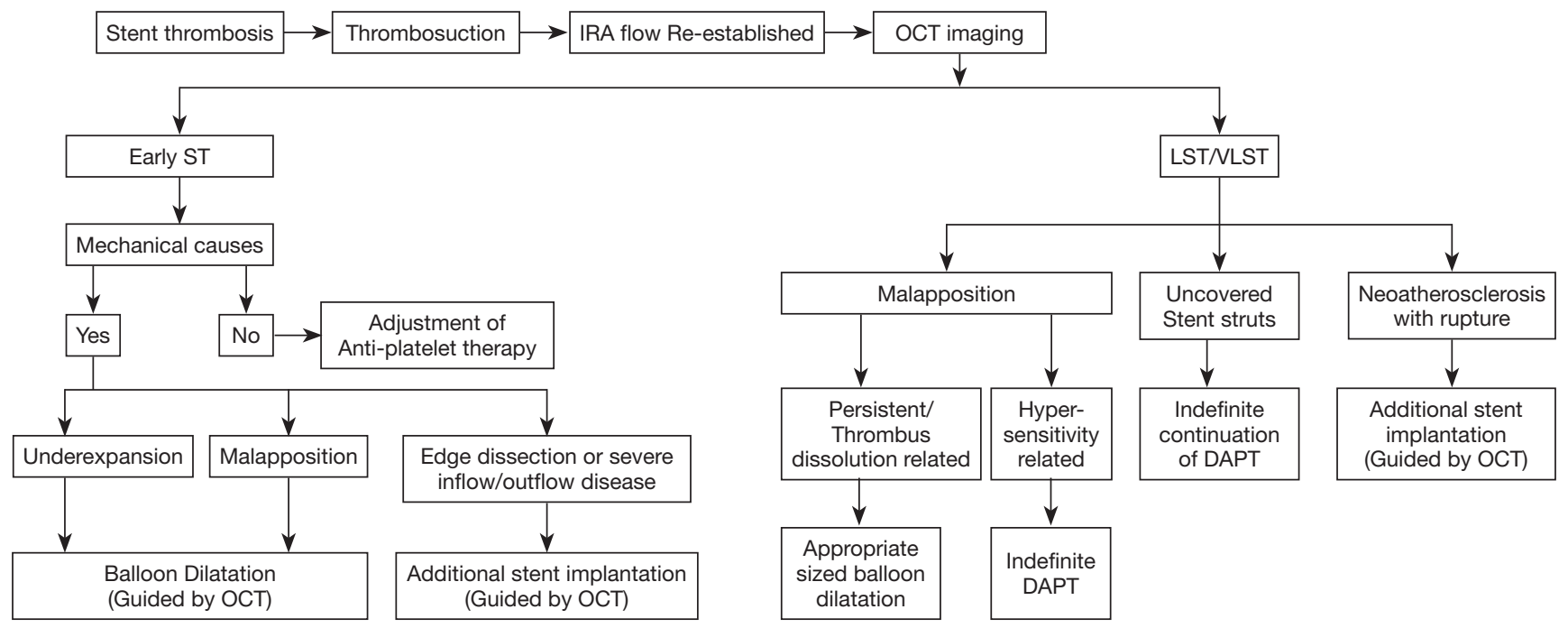

Figure 14 OCT-guided treatment of stent thrombosis. OCT, optical coherence tomography; IRA, infarct related artery; ST, stent thrombosis; LST, late stent thrombosis; VLST, very late stent thrombosis; DAPT, dual antiplatelet therapy. 


\section{Acknowledgments}

Funding: None.

\section{Footnote}

Provenance and Peer Review: This article was commissioned by the Guest Editor (Dennis Wong) for the series "Intracoronary Imaging" published in Cardiovascular Diagnosis and Therapy. The article was sent for external peer review organized by the Guest Editor and the editorial office.

Conflicts of Interest: Both authors have completed the ICMJE uniform disclosure forms (available at http://dx. doi. org/10. 21037/cdt-20-253). The series "Intracoronary Imaging" was commissioned by the editorial office without any funding or sponsorship. The authors have no other conflicts of interest to declare.

Ethical Statement: The authors are accountable for all aspects of the work in ensuring that questions related to the accuracy or integrity of any part of the work are appropriately investigated and resolved.

Open Access Statement: This is an Open Access article distributed in accordance with the Creative Commons Attribution-NonCommercial-NoDerivs 4.0 International License (CC BY-NC-ND 4.0), which permits the noncommercial replication and distribution of the article with the strict proviso that no changes or edits are made and the original work is properly cited (including links to both the formal publication through the relevant DOI and the license). See: https://creativecommons.org/licenses/by-nc-nd/4.0/.

\section{References}

1. DeRouen TA, Murray JA, Owen W. Variability in the analysis of coronary arteriograms. Circulation 1977;55:324-8.

2. Tearney GJ, Regar E, Akasaka T, et al. Consensus standards for acquisition, measurement, and reporting of intravascular optical coherence tomography studies: a report from the International Working Group for Intravascular Optical Coherence Tomography Standardization and Validation. J Am Coll Cardiol 2012;59:1058-72.

3. Jang IK. Cardiovascular OCT Imaging. 2nd edition. Springer, 2020.
4. van der Sijde JN, Guagliumi G, Sirbu V, et al. The OPTIS Integrated System: real-time, co-registration of angiography and optical coherence tomography. EuroIntervention 2016;12:855-60.

5. Xu C, Schmitt JM, Carlier SG, et al. Characterization of atherosclerosis plaques by measuring both backscattering and attenuation coefficients in optical coherence tomography. J Biomed Opt 2008;13:034003.

6. Yahagi K, Kolodgie FD, Otsuka F, et al. Pathophysiology of native coronary, vein graft, and in-stent atherosclerosis. Nat Rev Cardiol 2016;13:79-98.

7. Otsuka F, Joner M, Prati F, et al. Clinical classification of plaque morphology in coronary disease. Nat Rev Cardiol 2014;11:379-89.

8. Räber L, Radu MD, Mark BL. Atherosclerosis visualised with optimal coherence tomography. In: Radu MD, Räber L, Onuma Y, et al. editors. The clinical atlas of optical coherence tomography. 2nd edition. Toulouse, France: PCR Publishing, 2019.

9. Jia H, Abtahian F, Aguirre AD, et al. In vivo diagnosis of plaque erosion and calcified nodule in patients with acute coronary syndrome by intravascular optical coherence tomography. J Am Coll Cardiol 2013;62:1748-58.

10. Kang SJ, Nakano M, Virmani R, et al. OCT findings in patients with recanalization of organized thrombi in coronary arteries. JACC Cardiovasc Imaging 2012;5:725-32.

11. Yahagi K, Kolodgie FD, Virmani R. Epidemiology and pathophysiology of coronary artery disease. In: Grech E. editor. Practical Interventional Cardiology. Boca Raton: CRC Press, 2018:3-15.

12. Phipps JE, Houts T, Halaney, et al. Intravascular OCT Imaging Artifacts. In: Jang IK. editor. Cardiovascular OCT Imaging. 2nd edition. Springer International Publishing, 2020.:53-66

13. van Soest G, Regar E, Goderie TP, et al. Pitfalls in plaque characterization by OCT: image artifacts in native coronary arteries. JACC Cardiovasc Imaging 2011;4:810-3.

14. Elahi S, Mancuso JJ, Milner TE, et al. Sunflower artifact in OCT. JACC Cardiovasc Imaging 2011;4:1220-1.

15. Gonzalo N, Escaned J, Alfonso F, et al. Morphometric assessment of coronary stenosis relevance with optical coherence tomography: a comparison with fractional flow reserve and intravascular ultrasound. J Am Coll Cardiol 2012;59:1080-9.

16. Shiono Y, Kitabata H, Kubo T, et al. Optical coherence tomography-derived anatomical criteria for functionally 
significant coronary stenosis assessed by fractional flow reserve. Circ J 2012;76:2218-25.

17. Pawlowski T, Prati F, Kulawik T, et al. Optical coherence tomography criteria for defining functional severity of intermediate lesions: a comparative study with FFR. Int J Cardiovasc Imaging 2013;29:1685-91.

18. Reith S, Battermann S, Hellmich M, et al. Correlation between optical coherence tomography-derived intraluminal parameters and fractional flow reserve measurements in intermediate grade coronary lesions: a comparison between diabetic and non-diabetic patients. Clin Res Cardiol 2015;104:59-70.

19. Zafar H, Ullah I, Dinneen K, et al. Evaluation of hemodynamically severe coronary stenosis as determined by fractional flow reserve with frequency domain optical coherence tomography measured anatomical parameters. J Cardiol 2014;64:19-24.

20. Burzotta F, Nerla R, Hill J, et al. Correlation between frequency-domain optical coherence tomography and fractional flow reserve in angiographically-intermediate coronary lesions. Int J Cardiol 2018;253:55-60.

21. Burzotta F, Leone AM, Aurigemma C, et al. Fractional Flow Reserve or Optical Coherence Tomography to Guide Management of Angiographically Intermediate Coronary Stenosis: A Single-Center Trial. JACC Cardiovasc Interv 2020;13:49-58.

22. Yu W, Huang J, Jia D, et al. Diagnostic accuracy of intracoronary optical coherence tomography-derived fractional flow reserve for assessment of coronary stenosis severity. EuroIntervention 2019;15:189-97.

23. Sinclair H, Bourantas C, Bagnall A, et al. OCT for the identification of vulnerable plaque in acute coronary syndrome. JACC Cardiovasc Imaging 2015;8:198-209.

24. Vancraeynest D, Pasquet A, Roelants V, et al Imaging the vulnerable plaque. J Am Coll Cardiol 2011;57:1961-79.

25. Pu J, Mintz GS, Biro S, et al. Insights into echo-attenuated plaques, echolucent plaques, and plaques with spotty calcification: novel findings from comparisons among intravascular ultrasound, near-infrared spectroscopy, and pathological histology in 2,294 human coronary artery segments. J Am Coll Cardiol 2014;63:2220-33.

26. Stone GW, Maehara A, Lansky AJ, et al. A prospective natural-history study of coronary atherosclerosis. N Engl J Med 2011;364:226-35.

27. Uemura S, Ishigami K, Soeda T, et al. Thin-cap fibroatheroma and microchannel findings in optical coherence tomography correlate with subsequent progression of coronary atheromatous plaques. Eur Heart
J 2012;33:78-85.

28. Narula J, Kovacic JC. Putting TCFA in clinical perspective. J Am Coll Cardiol 2014;64:681-3.

29. Amabile N, Hammas S, Fradi S, et al. Intra-coronary thrombus evolution during acute coronary syndrome: regression assessment by serial optical coherence tomography analyses. Eur Heart J Cardiovasc Imaging 2015;16:433-40.

30. Opolski MP, Spiewak M, Marczak M, et al. Mechanisms of Myocardial Infarction in Patients With Nonobstructive Coronary Artery Disease: Results From the Optical Coherence Tomography Study. JACC Cardiovasc Imaging 2019;12:2210-21.

31. Tian J, Ren X, Vergallo R, et al. Distinct morphological features of ruptured culprit plaque for acute coronary events compared to those with silent rupture and thin-cap fibroatheroma: a combined optical coherence tomography and intravascular ultrasound study. J Am Coll Cardiol 2014;63:2209-16.

32. Johnson TW, Raber L, di Mario C, et al. Clinical use of intracoronary imaging. Part 2: acute coronary syndromes, ambiguous coronary angiography findings, and guiding interventional decision-making: an expert consensus document of the European Association of Percutaneous Cardiovascular Interventions. Eur Heart J 2019;40:2566-84.

33. Saia F, Komukai K, Capodanno D, et al. Eroded Versus Ruptured Plaques at the Culprit Site of STEMI: In Vivo Pathophysiological Features and Response to Primary PCI. JACC Cardiovasc Imaging 2015;8:566-75.

34. Higuma T, Soeda T, Abe N, et al. A Combined Optical Coherence Tomography and Intravascular Ultrasound Study on Plaque Rupture, Plaque Erosion, and Calcified Nodule in Patients With ST-Segment Elevation Myocardial Infarction: Incidence, Morphologic Characteristics, and Outcomes After Percutaneous Coronary Intervention. JACC Cardiovasc Interv 2015;8:1166-76.

35. Stone GW, Narula J. Emergence of plaque erosion as an important clinical entity. JACC Cardiovasc Imaging 2015;8:623-5.

36. Holmes DR, Jr., Lerman A, Moreno PR, et al. 3rd, Sharma SK. Diagnosis and management of STEMI arising from plaque erosion. JACC Cardiovasc Imaging 2013;6:290-6.

37. Partida RA, Libby P, Crea F, et al. Plaque erosion: a new in vivo diagnosis and a potential major shift in the management of patients with acute coronary syndromes. Eur Heart J 2018;39:2070-6. 
38. Jia H, Dai J, Hou J, et al. Effective anti-thrombotic therapy without stenting: intravascular optical coherence tomography-based management in plaque erosion (the EROSION study). Eur Heart J 2017;38:792-800.

39. Prati F, Uemura S, Souteyrand G, et al. OCT-based diagnosis and management of STEMI associated with intact fibrous cap. JACC Cardiovasc Imaging 2013;6:283-7.

40. Xing L, Yamamoto E, Sugiyama T, et al. EROSION Study (Effective Anti-Thrombotic Therapy Without Stenting: Intravascular Optical Coherence Tomography-Based Management in Plaque Erosion): A 1-Year Follow-Up Report. Circ Cardiovasc Interv 2017;10:e005860.

41. Alfonso F, Rivero F. Antithrombotic Therapy Alone for Plaque Erosion: Ready for a Paradigm Shift in Acute Coronary Syndromes?. Circ Cardiovasc Interv 2017;10:e006143.

42. Lee T, Mintz GS, Matsumura M, et al. Prevalence, Predictors, and Clinical Presentation of a Calcified Nodule as Assessed by Optical Coherence Tomography. JACC Cardiovasc Imaging 2017;10:883-91.

43. Alfonso F, Garcia-Guimaraes M, Bastante T, et al. Spontaneous coronary artery dissection: from expert consensus statements to evidence-based medicine. J Thorac Dis 2018;10:4602-8.

44. Hayes SN, Kim ESH, Saw J, et al. Spontaneous Coronary Artery Dissection: Current State of the Science: A Scientific Statement From the American Heart Association. Circulation 2018;137:e523-57.

45. Paulo M, Sandoval J, Lennie V, et al. Combined use of OCT and IVUS in spontaneous coronary artery dissection. JACC Cardiovasc Imaging 2013;6:830-2.

46. Terada K, Tanaka A, Katayama Y, et al. Role of Optical Coherence Tomography in the Diagnosis of Spontaneous Coronary Artery Dissection. International Cardiovascular Forum Journal 2019;14:3-7.

47. Yumoto K, Sasaki H, Aoki H, et al. Successful treatment of spontaneous coronary artery dissection with cutting balloon angioplasty as evaluated with optical coherence tomography. JACC Cardiovasc Interv 2014;7:817-9.

48. Parsa A, Saw J. OCT Imaging of SCAD and Differential Diagnosis. In: Jang IK. editor. Cardiovascular OCT Imaging. 2nd edition. Springer Nature, 2020:91-104.

49. Slavich M, Patel RS. Coronary artery spasm: Current knowledge and residual uncertainties. Int J Cardiol Heart Vasc 2016;10:47-53.

50. Tanaka A, Shimada K, Tearney GJ, et al. Conformational change in coronary artery structure assessed by optical coherence tomography in patients with vasospastic angina. J Am Coll Cardiol 2011;58:1608-13.

51. Shin ES, Ann SH, Singh GB, et al. OCT-Defined Morphological Characteristics of Coronary Artery Spasm Sites in Vasospastic Angina. JACC Cardiovasc Imaging 2015;8:1059-67.

52. Park HC, Shin JH, Jeong WK, et al. Comparison of morphologic findings obtained by optical coherence tomography in acute coronary syndrome caused by vasospasm and chronic stable variant angina. Int $\mathrm{J}$ Cardiovasc Imaging 2015;31:229-37.

53. Negishi Y, Ishii H, Suzuki S, et al. The combination assessment of lipid pool and thrombus by optical coherence tomography can predict the filter no-reflow in primary PCI for ST elevated myocardial infarction. Medicine (Baltimore) 2017;96:e9297.

54. Iakovou I, Ge L, Colombo A. Contemporary stent treatment of coronary bifurcations. J Am Coll Cardiol 2005;46:1446-55.

55. Lefevre T, Louvard Y, Morice MC, et al. Stenting of bifurcation lesions: classification, treatments, and results. Catheter Cardiovasc Interv 2000;49:274-83.

56. Holm NR, Adriaenssens T, Motreff P, et al. OCT for bifurcation stenting: what have we learned? EuroIntervention 2015;11 Suppl V:V64-70.

57. Watanabe $M$, Uemura $S$, Sugawara $Y$, et al. Side branch complication after a single-stent crossover technique: prediction with frequency domain optical coherence tomography. Coron Artery Dis 2014;25:321-9.

58. Okamura T, Onuma Y, Yamada J, et al. 3D optical coherence tomography: new insights into the process of optimal rewiring of side branches during bifurcational stenting. EuroIntervention 2014;10:907-15.

59. Nakao F. The impact of proximal cell rewiring compared with distal cell rewiring with single-stent deployment and subsequent kissing balloon inflation in the inadequate jailing of a Kaname stent on the side branch ostium. Cardiol J 2018;25:317-26.

60. Okamura T, Nagoshi R, Fujimura T, et al. Impact of guidewire recrossing point into stent jailed side branch for optimal kissing balloon dilatation: core lab 3D optical coherence tomography analysis. EuroIntervention 2018;13:e1785-93.

61. Viceconte N, Tyczynski P, Ferrante G, et al. Immediate results of bifurcational stenting assessed with optical coherence tomography. Catheter Cardiovasc Interv 
2013;81:519-28.

62. Holm NR, Andreasen LN, Walsh S, et al. Rational and design of the European randomized Optical Coherence Tomography Optimized Bifurcation Event Reduction Trial (OCTOBER). Am Heart J 2018;205:97-109.

63. Madhavan MV, Tarigopula M, Mintz GS, et al. Coronary artery calcification: pathogenesis and prognostic implications. J Am Coll Cardiol 2014;63:1703-14.

64. Chambers JW, Behrens AN, Martinsen BJ. Atherectomy Devices for the Treatment of Calcified Coronary Lesions. Interv Cardiol Clin 2016;5:143-51.

65. Mehanna E, Abbott JD, Bezerra HG. Optimizing Percutaneous Coronary Intervention in Calcified Lesions: Insights From Optical Coherence Tomography of Atherectomy. Circ Cardiovasc Interv 2018;11:e006813.

66. Kubo T, Shimamura K, Ino Y, et al. Superficial Calcium Fracture After PCI as Assessed by OCT. JACC Cardiovasc Imaging 2015;8:1228-9.

67. Maejima N, Hibi K, Saka K, et al. Relationship Between Thickness of Calcium on Optical Coherence Tomography and Crack Formation After Balloon Dilatation in Calcified Plaque Requiring Rotational Atherectomy. Circ J 2016;80:1413-9.

68. Fujino A, Mintz GS, Lee T, et al. Predictors of Calcium Fracture Derived From Balloon Angioplasty and its Effect on Stent Expansion Assessed by Optical Coherence Tomography. JACC Cardiovasc Interv 2018;11:1015-7.

69. Lund LH, Khush KK, Cherikh WS, et al. The Registry of the International Society for Heart and Lung Transplantation: Thirty-fourth Adult Heart Transplantation Report-2017; Focus Theme: Allograft ischemic time. J Heart Lung Transplant 2017;36:1037-46.

70. Ramzy D, Rao V, Brahm J, et al. Cardiac allograft vasculopathy: a review. Can J Surg 2005;48:319-27.

71. Hou J, Lv H, Jia H, et al. OCT assessment of allograft vasculopathy in heart transplant recipients. JACC Cardiovasc Imaging 2012;5:662-3.

72. Cassar A, Matsuo Y, Herrmann J, et al. Coronary atherosclerosis with vulnerable plaque and complicated lesions in transplant recipients: new insight into cardiac allograft vasculopathy by optical coherence tomography. Eur Heart J 2013;34:2610-7.

73. Clemmensen TS, Holm NR, Eiskjaer H, et al. Layered Fibrotic Plaques Are the Predominant Component in Cardiac Allograft Vasculopathy: Systematic Findings and Risk Stratification by OCT. JACC Cardiovasc Imaging 2017;10:773-84.

74. Clemmensen TS, Holm NR, Eiskjaer H, et al.
Detection of early changes in the coronary artery microstructure after heart transplantation: A prospective optical coherence tomography study. J Heart Lung Transplant 2018;37:486-95.

75. Raber L, Mintz GS, Koskinas KC, et al. Clinical use of intracoronary imaging. Part 1: guidance and optimization of coronary interventions. An expert consensus document of the European Association of Percutaneous Cardiovascular Interventions. Eur Heart J 2018;39:3281-300.

76. Torrado J, Buckley L, Duran A, et al. Restenosis, Stent Thrombosis, and Bleeding Complications: Navigating Between Scylla and Charybdis. J Am Coll Cardiol 2018;71:1676-95.

77. Adriaenssens T, Joner M, Godschalk TC, et al. Optical Coherence Tomography Findings in Patients With Coronary Stent Thrombosis: A Report of the PRESTIGE Consortium (Prevention of Late Stent Thrombosis by an Interdisciplinary Global European Effort). Circulation 2017;136:1007-21.

78. Souteyrand G, Amabile N, Mangin L, et al. Mechanisms of stent thrombosis analysed by optical coherence tomography: insights from the national PESTO French registry. Eur Heart J 2016;37:1208-16.

79. Soeda T, Uemura S, Park SJ, et al. Incidence and Clinical Significance of Poststent Optical Coherence Tomography Findings: One-Year Follow-Up Study From a Multicenter Registry. Circulation 2015;132:1020-9.

80. Lee SY, Shin DH, Kim JS, et al. Optical coherence tomographic observation of morphological features of neointimal tissue after drug-eluting stent implantation. Yonsei Med J 2014;5 5:944-52.

81. Yamamoto W, Fujii K, Otsuji S, et al. Optical coherence tomography characteristics of in-stent restenosis after drug-eluting stent implantation: a novel classification and its clinical significance. Heart Vessels 2020;35:38-45.

82. Lutter C, Mori H, Yahagi K, et al. Histopathological Differential Diagnosis of Optical Coherence Tomographic Image Interpretation After Stenting. JACC Cardiovasc Interv 2016;9:2511-23.

83. Lee JH, Jung HW, Kim JS, et al. Different Neointimal Pattern in Early vs. Late In-Stent Restenosis and Clinical Outcomes After Drug-Coated Balloon AngioplastyAn Optical Coherence Tomography Study. Circ J 2018;82:2745-52.

84. Alfonso F, Fernandez-Vina F, Medina M, et al. Neoatherosclerosis: the missing link between very late stent thrombosis and very late in-stent restenosis. J Am 
Coll Cardiol 2013;61:e155.

85. Otsuka F, Byrne RA, Yahagi K, et al. Neoatherosclerosis: overview of histopathologic findings and implications for intravascular imaging assessment. Eur Heart J 2015;36:2147-59.

86. Iliescu CA, Cilingiroglu M, Giza DE, et al. "Bringing on the light" in a complex clinical scenario: Optical coherence tomography-guided discontinuation of antiplatelet therapy in cancer patients with coronary artery disease (PROTECT-OCT registry). Am Heart J 2017;194:83-91.

87. Mariani J, Jr., Guedes C, Soares P, et al. Intravascular ultrasound guidance to minimize the use of iodine contrast in percutaneous coronary intervention: the MOZART

Cite this article as: Subban V, Raffel OC. Optical coherence tomography: fundamentals and clinical utility. Cardiovasc Diagn Ther 2020;10(5):1389-1414. doi: 10.21037/cdt-20-253
(Minimizing cOntrast utiliZation With IVUS Guidance in coRonary angioplas Ty) randomized controlled trial. JACC Cardiovasc Interv 2014;7:1287-93.

88. Azzalini L, Mitomo S, Hachinohe D, et al. Zero-Contrast Percutaneous Coronary Intervention Guided by DextranBased Optical Coherence Tomography. Can J Cardiol 2018;34:342.e1-342.e3.

89. Vasu N, Subban V, Ajit Mullasari S. Zero contrast optical coherence tomography-guided percutaneous coronary intervention for in-stent restenosis of the saphenous vein graft using a non-contrast flush medium. Indian Heart J 2018;70 Suppl 3:S492-5. 